OPEN ACCESS

Edited by:

Udo S. Gaipl,

University Hospital Erlangen, Germany

Reviewed by:

Yong Lu,

Wake Forest School of Medicine,

United States

Carlo Genova,

San Martino Hospital (IRCCS), Italy

*Correspondence:

Hong Zhu

zhuhonghuaxi@163.com

tThese authors have contributed equally to this work

Specialty section:

This article was submitted to Cancer Immunity and Immunotherapy,

a section of the journal Frontiers in Oncology

Received: 09 September 2020 Accepted: 15 February 2021 Published: 18 March 2021

Citation:

Hou W, Zhou X, Yi C and Zhu H (2021) Immune Check Point Inhibitors and Immune-Related Adverse Events in Small Cell Lung Cancer.

Front. Oncol. 11:604227. doi: 10.3389/fonc.2021.604227

\section{Immune Check Point Inhibitors and Immune-Related Adverse Events in Small Cell Lung Cancer}

\author{
Wanting Hou ${ }^{\dagger}$ Xiaohan Zhou ${ }^{\dagger}$, Cheng Yi and Hong Zhu* \\ Department of Medical Oncology, Cancer Center, West China Hospital, Sichuan University, Chengdu, China
}

Small cell lung cancer (SCLC) is a malignant solid tumor. In recent years, although immune check point inhibitors (ICls) have achieved important advances in the treatment of SCLC, immune-related adverse events (irAEs) have occurred at the same time during the therapeutic period. Some irAEs lead to dose reduction or treatment rejection. The immune microenvironment of SCLC is complicated, therefore, understanding irAEs associated with ICls is of great importance and necessity for the clinical management of SCLC. However, the lack of comprehensive understanding of irAEs in patients with SCLC remains remarkable. This review aims to provide an up-to-date overview of ICls and their associated irAEs in patients with SCLC based on present clinical data.

Keywords: small cell lung cancer, immune check point inhibitors (ICIs), immune-related adverse events (irAEs), programmed cell death protein 1 (PD-1), programmed cell death ligand protein 1 (PD-L1), cytotoxic T lymphocyte-associated antigen 4 (CTLA-4)

\section{INTRODUCTION}

Lung cancer has jeopardized the health of millions of people worldwide (1). Histologically, lung cancer is usually classified into non-small cell lung cancer (NSCLC) and small cell lung cancer (SCLC). Although NSCLC is diagnosed more often, SCLC is much more likely to be associated with a worse prognosis. SCLC is characterized by rapid growth, early metastasis, and frequent relapse (2) and its diagnoses are further sub-divided into limited-stage SCLC (LS-SCLC) or extensive-stage SCLC (ES-SCLC). LS-SCLC is diagnosed when the diseased region is confined to one hemithorax within a tolerable radiation field, while ES-SCLC is diagnosed when the disease has spread beyond one hemithorax. The prognosis of patients with LS-SCLC is better than those with ES-SCLC, the survival time of patients with LS-SCLC ranges from 15 to 20 months and their 5-year survival rate is $\sim 20-25 \%$. In comparison, the survival time of patients with ES-SCLC ranges from 8 to 13 months, with a 5 -year survival rate of only about $2 \%(3,4)$. Unfortunately, $70 \%$ of SCLC patients are diagnosed with ES-SCLC (5).

Compared with NSCLC, SCLC's effective therapy regimens are limited. In the past, platinum-based chemotherapy has been the cornerstone of the SCLC therapeutic landscape. The overall response rate (ORR) of ES-SCLC patients receiving first-line chemotherapy is $\sim 67 \%$, with most patients showing resistance to chemotherapy within a short time and overall survival (OS) of $<1$ year. Less than $30 \%$ of patients are diagnosed with LS-SCLC and they respond well to chemotherapy (ORR: 82-87\%). However, preventing relapse and progression is still challenging (6). At present, second-line treatment options for patients with relapsed SCLC are limited. In 2007, the FDA approved topotecan as a second-line treatment option for SCLC. The response rate to topotecan of patients who have relapsed is $20-25 \%$, with a 1-year survival rate of $10-30 \%(7,8)$. Over the years, there are a few new explorations in the therapeutic landscape of SCLC. The success 
of immunotherapy for NSCLC is great inspiration for SCLC therapy. Several clinical trials on monoclonal antibodies targeting programmed cell death ligand protein 1 (PD-L1), programmed cell death protein 1 (PD-1), and cytotoxic T-lymphocyteassociated antigen 4 (CTLA-4) have been or are currently being conducted for SCLC. The response rate to immunotherapy is lower for SCLC than for other tumors such as NSCLC and melanoma, as a possible result of the lack of biomarkers for choosing beneficial populations. Nevertheless, immunotherapy still brings breakthroughs for SCLC therapy. Based on the encouraging results of CheckMate032, KEYNOTE028, and KEYNOTE158, FDA approved nivolumab and pembrolizumab monotherapy as third or later line for the treatment of patients with relapsed SCLC. Compared with chemotherapy alone, the PD-L1 inhibitors atezolizumab and durvalumab plus the chemotherapy agent's platinum and etoposide have demonstrated prolonged OS among ES-SCLC patients. In addition, these two PD-L1 inhibitors plus chemotherapy have been approved by FDA as first-line therapy for ES-SCLC (Table 1). Recently, several meta-analyses analyzed different firstline treatments for ES-SCLC patients and demonstrated that PDL1 inhibitors durvalumab and atezolizumab plus etoposide-based chemotherapy may be the best choice as first-line therapy for ES-SCLC patients (9-11).

Meanwhile, there is increasing concern regarding immunerelated adverse events (irAEs) of ICIs (12-14). The appearance of some irAEs have beenshown to be related to the efficacy of ICI agents in patients with NSCLC and melanoma $(15,16)$, but this relationship has not been established in patients with SCLC. SCLC is characterized by complex immunophenotypes, and autoimmune-related paraneoplastic syndromes are commonly reported among SCLC patients (17). Understanding irAEs of ICIs is crucial for the clinical management of SCLC and for further improvement of the immunotherapeutic approach to SCLC, but few studies have focused on irAEs in SCLC recently. Therefore, in this article, we present an up-to-date review of ICIs and irAEs in SCLC based on data from present clinical trials.

\section{Mechanism of ICls and irAEs}

Under normal conditions, immune inhibitor molecules such as CTLA-4 and PD-1/PD-L1 function as negative regulators and maintain the balance of the immune system. CTLA- 4 and CD28 are commonly expressed on the surface of T cells, where they compete with the same binding sites as CD80/CD86 on the surface of antigen-presenting cells (APCs). CD28 combines with $\mathrm{CD} 80 / \mathrm{CD} 86$ to provide an activation signal for $\mathrm{T}$ cells. The combination of CTLA-4 and CD80/CD86 impedes T-cell activation and downregulates $\mathrm{T}$-cell responses $(18,19)$. Unlike CTLA-4, PD-1 is expressed on the surface $\mathrm{T}$ cells or other immune cells, and its ligand, PD-L1, is expressed on the surface of APCs and other immune cells. Tumor cells can also express PD-L1, and PD-1 and PD-L1 combine on the surface of tumor cells, leading to downregulation of the T-cell response, and helping tumor cells to escape from the host immune response (20, 21). Tumor cells upregulate these immune inhibitor molecules to evade the immune system, resulting in tumor initiation, progression, and metastasis. The ICI blockade of PD1/PD-L1 or CTLA-4 activates the body's antitumor immunity (Figure 1).

IrAEs also occur in this context. IrAEs are generally considered to be related to the damage of normal tissues, which results from immunotherapy, and immune tolerance is affected by ICIs, resulting in activated $\mathrm{T}$ cells targeting non-tumor antigens or self-peptides. IrAEs affect almost all organs. The most common irAEs include rash, pruritus, colitis, hypothyroidism, hyperthyroidism, and pneumonitis. The precise pathophysiology of irAEs is still undefined. Existing studies suggest that autoantibodies play an important role in irAEs. Some autoantibodies may have cross-reactivity with antibodies during immunotherapy (22). One example is vitiligo, which is caused by an autoantibody attack on melanocytes, and it is also frequently observed among melanoma patients who have received ICI therapy (23). In addition, some cytokines may be involved in irAEs (22). For instance, the levels of the interleukin17 were obviously higher in patients with ipilimumab-related colitis (24). Host factors, intestinal microbiota, genetic risk factors, and specific antigen exposures may all be involved in irAEs (25). Viruses or co-administered drugs can also provoke irAEs (26). CTLA-4 and PD1/PD-L1 inhibitors usually display different irAEs. Reportedly, the occurrence rate of diarrhea and colitis in patients administered CTLA- 4 is higher than that in those administered PD-1/PD-L1 antibodies. Thyroiditis and pneumonitis are more commonly observed in patients who received $\mathrm{PD}-1 / \mathrm{PD}-\mathrm{L} 1$ inhibitors. Compared with ICI monotherapy, combinations of ICIs increase the risk of irAEs. Tumors also influence irAEs, with colitis and skin irAEs being more common in patients treated with ICIs for melanoma and pneumonitis occurring more frequently in lung cancer patients treated with ICIs (25). Accurately recognizing irAEs and closely following up patients who have used ICIs are essential parts of immunotherapy.

\section{The Immune Characteristics and Response to ICls of SCLC}

The efficacy of immunotherapy is largely determined by the internal immune microenvironment of the tumor (27). However, it is widely regarded that SCLC has a unique and complex immune microenvironment.

Consistently, previous studies regard SCLC as a kind of immunogenic cancer) One of the hallmarks of SCLC is high tumor mutational burdens (TMBs) $(28,29)$, which are usually used as a predictor of ICI efficacy for many cancer types, including NSCLC (30) and melanoma (31). Reportedly, High TMBs could influence further neo-antigens to activate the immune system (32). Moreover, paraneoplastic syndromes are commonly observed in SCLC patients and are mediated by autoantibodies. Evidence shows that SCLC patients with neurologic paraneoplastic syndromes present with better prognosis $(33,34)$. While immunosuppression does exist in SCLC, lower expression of class I major histocompatibility antigens, tumor-infiltrating lymphocytes, and PD-L1 have also been reported in some SCLC patients (3). 
TABLE 1 | FDA approved ICls in SCLC.

\begin{tabular}{|c|c|c|c|c|c|}
\hline Agent & Target & Therapy line & Patients & Approve time & Based clinical trail \\
\hline Nivolumab & PD-1 & Third line or later line & Relapsed-SCLC & 2018.08 & CheckMate032 \\
\hline Atezolizumab+ platinum-etoposide & PD-L1 & First line & ES-SCLC & 2019.03 & IMpower133 \\
\hline Pembrolizumab & PD-1 & Third line or later line & Relapsed-SCLC & 2019.06 & KEYNOTE028 and KEYNOTE158 \\
\hline Durvalumab+ platinum-etoposide & PD-L1 & First line & ES-SCLC & 2020.03 & CASPIAN \\
\hline
\end{tabular}

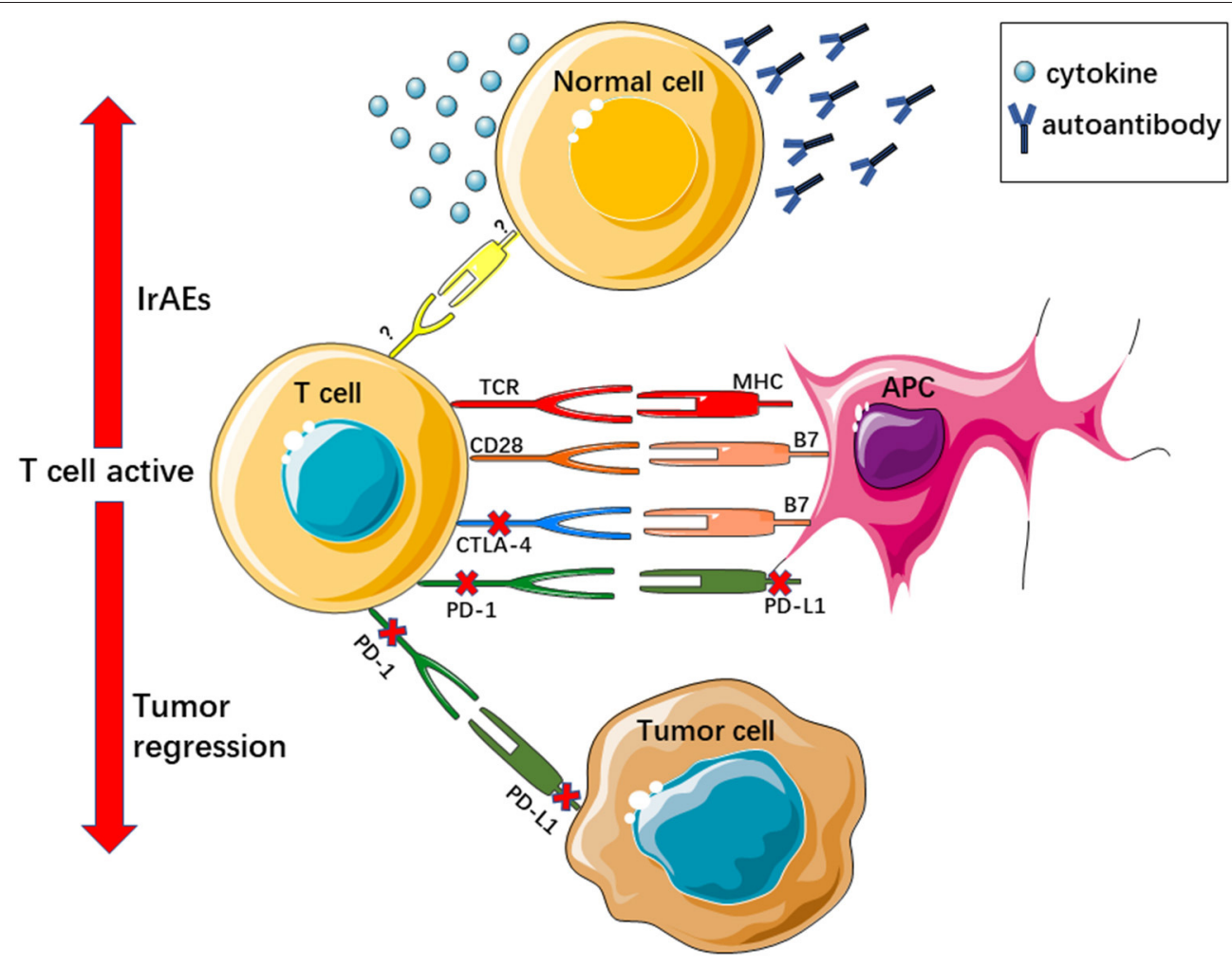

FIGURE 1 | ICls reactive T cell active regress tumor growth and evoke irAEs. TCR, T cell receptor; MHC, major histocompatibility complex.

The response rate to ICIs, particularly ICI monotherapy, was lower in most patients with SCLC than in those with other tumors. PD-1 antibody nivolumab monotherapy did not present any advantage in the improvement of OS or progression-free survival (PFS) as second-line treatment setting for relapsed SCLC according to the Checkmate 331 study (35). Moreover, the PD-L1 inhibitor durvalumab monotherapy for relapsed SCLC patients presented a confirmed ORR of only 9.5\% (36).

Furthermore, the overall low response to ICIs of SCLC patients may be due to the lack of biomarkers. In the past years, relatively few tumor specimens for SCLC have been available. In addition, immune heterogeneities have been found among SCLC patients; in particular, tumor cell PD-L1 expression was different among various studies (37). No reliable biomarkers have yet been confirmed in terms of the population of SCLC patients who will benefit from ICI immunotherapy, which could influence the ORR. Recently, comprehensive research related to SCLC's biomarkers in the tumor stromal cell or in the blood are largely being conducted $(38,39)$. However, the findings still need to be assessed in large samples. Nevertheless, compared with single chemotherapy, PD-L1 inhibitor with chemotherapy for SCLC patients presented beneficial OS (A et al., 2019) (40), and there is increasing evidence that shows that combination therapy, such as combined with chemotherapy, radiotherapy, and other targeted therapy, may overcome the low response to ICI among SCLC patients $(38,41,42)$.

\section{PD-1 Inhibitors and irAEs in SCLC}

Three PD-1 inhibitors, namely nivolumab, pembrolizumab, and tislelizumab, have been investigated in SCLC clinical trials as monotherapy or in combination chemotherapy.

In the nivolumab monotherapy arm of the Checkmate 032 study, the SCLC cohort comprised 109 chemotherapy-refractory SCLC patients who received nivolumab $(3 \mathrm{mg} / \mathrm{kg})$ as third-line or later-line therapy. After a median follow-up time of 28.3 months, the reported ORR was $11.9 \%$. The median duration of response (DOR) was 17.9 months (range: 3.0-42.1). The median PFS and median OS were 1.4 months (95\% CI: 1.3-1.6) and 5.6 months 
(95\% CI: 3.1-6.8), respectively. Approximately 55\% of patients in the nivolumab monotherapy group experienced treatmentrelated adverse events (TRAEs), and grade 3 to 4 TRAEs were reported in $11.9 \%$ of patients. Most irAEs reported in the study, including reactions of the skin $(21.1 \%)$, endocrine system $(9.2 \%)$, gastrointestinal tract $(6.4 \%)$, hepatic system $(4.6 \%)$, pulmonary system (1.8\%), renal system ( $0.9 \%)$, and hypersensitivity/infusion reactions (3.7\%), were mild (grade 1 to 2 ). Grade 3 to 4 irAEs included pneumonitis (1.8\%), rash (0.9\%), and aspartate aminotransferase $(0.9 \%)$. One patient experienced grade 3-4 treatment-related encephalitis, and one death was reported due to treatment-related pneumonitis (43). Based on these promising results, the FDA approved nivolumab as third-line or later-line therapy for relapsed SCLC.

Nivolumab as second-line treatment for relapsed SCLC was evaluated in Checkmate 331 (NCT02481830). The preliminary results showed no significant differences in OS, PFS, ORR, and DOR during nivolumab monotherapy. The TRAEs were reported to be lower in the nivolumab group (grade $3=55 \%$, grade $4=$ $14 \%$ ) than in the chemotherapy group (grade $3=90 \%$, grade $4=73 \%$ ) (35). Further details of TRAEs/irAEs associated with Checkmate 331 are still pending.

Pembrolizumab was another immunoglobulin G4 (IgG4) monoclonal antibody of PD-1 (44). Pembrolizumab first showed efficacy among the PD-L1-positive SCLC patients of the Keynote 028 study, with promising primary end point ORR $(33.3 \%$, 95\% CI: $15.6-55.3 \%$ ) and secondary end points (median DOR, median PFS, and median OS). The median DOR observed was 19.4 months, and the observed median PFS and median OS were 1.9 months and 9.7 months, respectively. TRAEs occurred in $66.7 \%$ of patients, and the most common events include arthralgia (16.6\%), asthenia (16.6\%), rash (16.7\%), diarrhea (12.5\%), and fatigue (12.5\%). Two patients (8.3\%) experienced treatment-related grade 3 to 5 AEs (one grade 3 bilirubin elevation and one grade 5 colitis/intestinal ischemia) (45).

Then, the Keynote 158 study enrolled 107 patients with SCLC (14\% were PD-L1 positive) who received $200 \mathrm{mg}$ of pembrolizumab treatment. Reportedly, the overall ORR was $18.7 \%$. Significantly, difference was found in the ORR, median PFS, and median OS of the PD-L1-positive and PD-L1-negative cohorts. The ORR, median PFS, and median OS in the PDL1-positive cohort presented superiority to that of the PD-L1negative cohort. TRAEs were reported in $60 \%$ of all enrolled patients, with $12 \%$ of patients experiencing grade 3 to 4 TRAEs. Additionally, a total of $33 \%$ of patients experienced irAEs, with $5 \%$ of patients experiencing (suffering) grade 3 4 irAEs. The most common irAEs were hypothyroidism and hyperthyroidism, which occurred in $12 \%$ and $7 \%$ of the patients, respectively. Severe skin reactions were reported in 3\% patients. Adrenal insufficiency, nephritis, pneumonitis and pancreatitis were all reported in $2 \%$ of the patients (46). In the according to subsequent pooled analysis of Keynote 028 and Keynote 158, the ORR was $19.3 \%$ (95\% CI: 11.4-29.4) and the median time to response was 2.1 months (range: 1.7-4.1). The median PFS and median OS was 2.0 months (95\% CI: 1.9-3.4) and 7.7 months (95\% CI: 5.2-10.1), respectively. TRAEs occurred in $61.4 \%$ of the patients, with $7.2 \%$ of patients experiencing grade 3 TRAEs. IrAEs were considered to have occurred in $24.1 \%$ of patients, with the most common events being hypothyroidism, hyperthyroidism, and infusion reactions. Grade 3 irAEs occurred in $6.0 \%$ patients, including colitis, adrenal insufficiency, pancreatitis, and pneumonitis. Most of the irAEs could be alleviate by systemic corticosteroid treatment (47). Based on these promising results, pembrolizumab was also approved as third-line or later-line therapy for relapsed SCLC.

Regarding maintenance therapy, pembrolizumab monotherapy did not show any improvement in median PFS for patients with ES-SCLC (48). The median PFS of 45 enrolled patients was 1.4 months (95\% CI: 1.3-2.8), and the median OS was 9.6 months (95\% CI: 7.0-12). Higher median PFS and higher median OS were observed in patients with tumor stromal expressing PD-L1. Most reported TRAEs were mild (grade 2 or lower). Three adverse events, including rash (18\%), hypothyroidism (9\%), and type I diabetes mellitus with diabetic ketoacidosis (11\%), were considered asirAEs.

Pembrolizumab was well tolerated during consolidation thoracic radiotherapy in a combination setting. In a singleinstitution phase I trial, after induction chemotherapy, 33 ESSCLC patientwere treated with 45 Gy thoracic radiotherapy plus pembrolizumab. No dose-limiting toxicities were observed in the first 35 days, and the median PFS and OS were 6.1 months and 8.4 months, respectively. No grade 4 or 5 treatment-related toxicities were reported. TRAEs occurred in $6 \%$ of patients, but the investigator considered these were unlikely to be related to treatment (49). Furthermore, pembrolizumab combined with concurrent chemoradiation therapy were assessed in LS-SCLC patients, with the median follow-up time of 23.1 months, and the reported median PFS and median OS were 19.7 months and 39.5 months, respectively. Most TRAEs were mild; only one grade 4 respiratory failure and two grade 4 neutropenia were reported. Fatigue, dysphagia, dyspnea, and anemia were the most common grade 1-2 TRAEs. Conversely, neutropenia and anemia were the most common grade 3 TRAEs. Treatment-related pneumonitis was reported in $15 \%$ of patients (49).

In the NCT02551432 study, it was reported that the pembrolizumab plus paclitaxel as second-line therapy in relapsed or refractory SCLC patients were not inferior to the traditional second-line chemotherapy. The reported ORR was $23.1 \%(95 \%$ CI: $6.9-39.3 \%)$. The median PFS and median OS were 5.0 months (95\% CI: 2.7-6.7) and 9.1 months (95\% CI: 6.5-15.0), respectively. All enrolled patients experienced adverse events., with $\sim 46 \%$ grade 3 or 4 adverse events were reported, including febrile neutropenia, neutropenia, asthenia, hyponatremia, and type I diabetes (50).

Recently, combination of pembrolizumab plus etoposide/platinum (EP) as the first-line therapeutic regimens for ES-SCLC patients were also evaluated in the Keynote 604 study. The results indicate that pembrolizumab plus EP significantly improved PFS, but the significance threshold for OS was not reached. A total of $24.7 \%$ irAEs were reported in the pembrolizumab plus chemotherapy group, and $10.3 \%$ of irAEs were reported in the chemotherapy group. The most common irAEs were hypothyroidism, hyperthyroidism, and pneumonitis. Grade 3 irAEs reported in the pembrolizumab 
plus chemotherapy group were $7.2 \%$ vs. the $0.9 \%$ in the chemotherapy group. The most common grade 3 irAEs were severe skin reactions $(1.8 \%)$, pneumonitis $(1.3 \%)$, and hepatitis (1.3\%) (51).

Tislelizumab is, a PD-1 antibody with high affinity and specificity, was explored in the first-line SCLC treatment setting in a phase II study (NCT03432598). 17 Chinese ES-SCLC patients were treated with tislelizumab $(200 \mathrm{mg})$ plus etoposide and platinum. The ORR was $77 \%$ (95\% CI: 50.1-93.2). The median PFS and median OS in the SCLC cohort was 6.9 months and 15.6 months.IrAEs in the SCLC cohort included thyroid disorders (29.4\%), pneumonitis (5.9\%), and type 1 diabetes mellitus (5.9\%) (52).

\section{PD-L1 Inhibitors and irAEs in SCLC}

PD-L1 inhibitor atezolizumab and durvalumab as combination agents with platinum-etoposide indicated a promising profile as first-line therapy for ES-SCLC patients.

NCT01375842, a first phase Ia study, assess atezolizumab's single agent clinical activity in patients with ES-SCLC. The study enrolled 17 patients, and after 6.7 months of follow-up, the confirmed ORR was 6\%. The reported median PFS and median OS were 1.5 months and 5.9 months, respectively. Most patients (65\%) experienced various grades of TRAEs, with the most common being fatigue (24\%). One grade 3 pneumonitis and one grade 5 hepatic failure were reported (53).

The IMpower133 trial further evaluated atezolizumab plus chemotherapy as first-line treatment option for ES-SCLC patients. The median OS and median PFS in the combined therapy group were both longer than in monotherapy group. The secondary end points ORR and DOR were similar between the two groups. IrAEs occurred in $39.9 \%$ of patients in the atezolizumab group and in $24.5 \%$ of patients in the placebo group. The most common irAEs included rash (18.7 vs. $10.2 \%$ ), hypothyroidism ( 12.6 vs. $0.5 \%$ ), hepatitis ( 7.1 vs. $4.6 \%$ ), infusion-related reactions (5.6 vs. 5.1\%), hyperthyroidism (5.6 vs. $2.6 \%$ ), pneumonitis (2.0 vs. $2.6 \%$ ), and colitis (1.5 vs. $0 \%)$. The grade 3-4 irAEs reported in the atezolizumab group included rash $(2.0 \%)$, hepatitis $(1.5 \%)$, infusion-related reaction (2.0\%), pneumonitis $(0.5 \%)$, colitis $(1.0 \%)$, pancreatitis $(0.5 \%)$, rhabdomyolysis $(0.5 \%)$, nephritis $(0.5 \%)$, and Guillain-Barre syndrome $(0.5 \%)$ (54). A following study further presented the safety and tolo data of the induction and maintenance phases of the IMpower 133 trial. The results were similar to those that had previously been reported. IrAEs were more frequently reported in the atezolizumab arm during both induction therapy and maintenance therapy, with rash and hypothyroidism being the most common irAEs (40).

In contrast, atezolizumab monotherapy failed to demonstrate clinic efficacy for relapsed SCLC patients in second line management setting in the non-comparative phase II IFCT-1603 study. The ORR of the atezolizumab group was only $2.3 \%$, and the median PFS of the chemotherapy group was longer than that of the atezolizumab group. Difference in median OS was observed between the two groups. IrAEs in the atezolizumab group included hepatitis (4.2\%), colitis (4.2\%), arthralgia (6.3\%), dysthyroidism (4.2\%), musculoskeletal and connective tissue disorders (12.5\%), and gastrointestinal disorders (18.8\%). Most of the irAEs were mild (grade 1 or 2) (55).

In March 2020, another anti-PD-L1 antibody, durvalumab, in combination with platinum-etoposide was approved by the FDA as first-line therapy of ES-SCLC patients based on the findings of the CASPIAN trial (NCT03043872). The CASPIAN study was designed as an open-label, phase 3 trial to assess durvalumab with or without tremelimumab in combination with platinum-etoposide as first-line treatment for ES-SCLC patients. Firstly, the result of durvalumab plus platinum-etoposide group and the platinum-etoposide group have been published in the planned interim analysis. The reported median OS in the durvalumab plus platinum-etoposide group was slightly longer than the platinum-etoposide group (13.0 months vs. 10.3 months, respectively). The median PFS and 6-month PFS was similar between the two groups. The 12-month PFS rates and 18-month OS rates were higher in the durvalumab plus platinum-etoposide arm. The confirmed ORR in the two groups was $68 \%$ and $58 \%$, respectively. Reported TRAEs were similar between the two groups ( 89 and $90 \%$, respectively). The grade 3-4 TRAEs were similar between the chemotherapy group and in the durvalumab plus chemotherapy group (both are 62\%). IrAEs were experienced by $20 \%$ of patients in the durvalumab plus chemotherapy group, with only $5 \%$ of patients experiencing grade 3 or 4 irAEs. Only $3 \%$ of patients in the chemotherapy group experienced irAEs and the occurrence rate of grade 3 or 4 irAEs was $<1 \%$. The most common irAEs were hypothyroid events ( 9 vs. $1 \%$ ), hyperthyroid events (5 vs. $0 \%$ ), pneumonitis (3 vs. $1 \%$ ), hepatic events (3 vs. $0 \%$ ), dermatitis/rash ( 2 vs. $1 \%$ ), and diarrhea/colitis ( 2 vs. $<1 \%$ ). Thyroiditis and type 1 diabetes mellitus only occurred in the durvalumab plus chemotherapy group. Other rare irAEs included one patient in the durvalumab plus chemotherapy group who experienced adrenal insufficiency, and one patient in the durvalumab plus chemotherapy group who experienced grade 3 or 4 pancreatic events. Grade 3 or 4 irAEs in the durvalumab plus chemotherapy group included pneumonitis (1\%), hepatic events $(2 \%)$, diarrhea/colitis $(<1 \%)$, type 1 diabetes mellitus $(2 \%)$, and pancreatic events $(<1 \%)$. Deaths due to irAEs occurred in $<1 \%$ of patients in each group. One therapy-related hepatotoxicity caused death in the durvalumab plus chemotherapy group and one therapy-related pneumonitis caused death in the platinum-etoposide group (56).

Similarly, the durvalumab monotherapy failed for relapsed SCLC patients. The confirmed ORR was only 9.5\% (95\% CI: 1.2-30.4). The median PFS and OS were 1.5 months (95\% CI: $0.9-1.8$ ) and 4.8 months (95\% CI: $1.3-10.4)$. The 12 -month OS rate was $27.6 \%$ (95\% CI: $10.2-48.4$ ). A total of $33.3 \%$ of patients had TRAEs, all grade 1 or 2. Nausea, fatigue, and maculo-papular rash were the most commonly reported TRAEs. No TRAEs led to discontinuation or death (36).

\section{CTLA-4 Inhibitors and irAEs in SCLC}

CA184-041 (NCT00527735) was the first phase II study exploring the clinical efficiency and tolerability of the CTLA-4 inhibitor ipilimumab in ES-SCLC patients. Chemotherapy-naïve ESSCLC patients were randomized to receive paclitaxel/carboplatin with either placebo, concurrent ipilimumab (ipilimumab plus 
paclitaxel/carboplatin followed by maintenance treatment with ipilimumab), or phased ipilimumab (paclitaxel/carboplatin administered before ipilimumab, followed by maintenance treatment with ipilimumab). Prolonged immune-related PFS (irPFS) was only reported in the phased ipilimumab group. Nonsignificant improvement in PFS and OS was observed in both the ipilimumab groups. The grade $3 / 4$ TRAEs were more common in the ipilimumab-containing arms. The most common irAEs were related to skin events (rash and pruritus), gastrointestinal events (diarrhea), and liver function (increases in alanine aminotransferase and aspartate aminotransferase), both of which occurred more frequently in both the concurrent ipilimumab and the phased ipilimumab groups. Most grade 3/4 irAEs could be managed well after follow-up or systemic corticosteroid treatment. Thus, phased ipilimumab demonstrated both efficacy and safety in previously untreated ES-SCLC patients in this clinical study (57).

In another phase II open-label study (NCT01331525), 42 chemotherapy-naïve ES-SCLC patients were treated with six cycles of carboplatin and etoposide plus ipilimumab. The study did not meet the primary endpoint (1-year PFS). The median PFS and median OS were 6.9 months and 17.0 months, respectively. In total, $69.2 \%$ of patients experienced serious irAEs ( $\geq$ grade 3 ), with the most frequent irAEs being diarrhea and skin rash. Serious ipilimumab-related neurological adverse events (grade 3 or higher) were reported in $7.6 \%$ of patients. Moreover, five deaths related to ipilimumab occurred; two of which were reported shortly after treatment (cardiac arrest, neutropenic sepsis) and three occurred 4-5 months after the last treatment (pneumonia, autoimmune encephalitis, and sepsis). This study additionally presented an association between improved outcomes and baseline autoimmunity of the therapy (58).

The phase III study CA184-156 (NCT01450761) investigated the efficacy and safety of ipilimumab plus etoposide and platinum for newly diagnosed ES-SCLC patients. The results showed that the addition of ipilimumab to chemotherapy did not present a survival benefit in ES-SCLC patients. The median OS and median PFS was found to be similar between the chemotherapy plus ipilimumab group and the chemotherapy plus placebo group. Patients receiving ipilimumab had more TRAEs, which required discontinuation of therapy (18 vs. $2 \%$ ). Gastrointestinal and skinrelated AEs were the most common irAEs (34 vs. 29\%). The other irAEs that presented in more than $5 \%$ of patients were diarrhea (25 vs. $10 \%$ ), rash (19 vs. $3 \%$ ), pruritus (12 vs. $2 \%$ ), colitis (6 vs. $1 \%$ ), alopecia ( 5 vs. $7 \%$ ), endocrine irAEs (10 vs. $2 \%$ ), and peripheral sensory neuropathy (2 vs. $1 \%$ ). Moreover, $76 \%$ of grade 2-4 irAEs were completely resolved. Neurologic events required more time (28.9 weeks) to resolve compared with other irAEs (59).

\section{Double Check Point Inhibitors and irAEs in SCLC}

Different from PD-1/PD-L1, CTLA-4 activates $\mathrm{T}$ cells in the early stage. In theory, the combination of these two inhibitors would be more effective than either of them alone. Previously, the combination of double checkpoint inhibitors has shown survival benefits in some solid tumors, such as advanced melanoma and relapsed malignant pleural mesothelioma $(60,61)$.

Some clinical trials of ICI combinations in SCLC patients have also been reported (62). In the Checkmate 451 study, ESSCLC patients were randomly receiving nivolumab $(1 \mathrm{mg} / \mathrm{kg})$ plus ipilimumab (3 mg/kg), nivolumab $(240 \mathrm{mg})$ as a single agent, or placebo. Neither nivolumab alone or in combination with ipilimumab significantly improved OS compared with placebo, and $86 \%$ of patients receiving nivolumab plus ipilimumab experienced adverse events. Toxicity-induced discontinuation of therapy was reportedly higher in the combination group than in the nivolumab monotherapy and placebo groups (32\% vs. $9 \%$ vs. $1 \%$, respectively). Moreover, the deaths of seven patients that were related to the treatment were reported in the nivolumab plus ipilimumab group (63).

In the Checkmate 032 study, eligible SCLC patients (both limited- and extensive-stage) were treated with nivolumab monotherapy $(3 \mathrm{mg} / \mathrm{kg}$ ) or nivolumab combined ipilimumab (nivolumab $1 \mathrm{mg} / \mathrm{kg}$ plus ipilimumab $1 \mathrm{mg} / \mathrm{kg}$, nivolumab 1 $\mathrm{mg} / \mathrm{kg}$ plus ipilimumab $3 \mathrm{mg} / \mathrm{kg}$, nivolumab $3 \mathrm{mg} / \mathrm{kg}$ plus ipilimumab $1 \mathrm{mg} / \mathrm{kg}$ ). The results showed that nivolumab plus ipilimumab significantly improved ORR in SCLC patients. In addition, the median OS was improved in the combination group, but the median PFS of the nivolumab $(3 \mathrm{mg} / \mathrm{kg})$ plus ipilimumab $(1 \mathrm{mg} / \mathrm{kg})$ group was similar to that of the nivolumab monotherapy group. Adverse events, including all grades of adverse events, were also higher in the combination group. Increased lipase and diarrhea were the most commonly reported grade 3-4 TRAEs. The incidence of discontinued treatment due to TRAEs was higher in the nivolumab (1 $\mathrm{mg} / \mathrm{kg}$ ) plus ipilimumab (3 $\mathrm{mg} / \mathrm{kg}$ ) cohort, whereas that of discontinued treatment due to TRAEs was similar in the nivolumab monotherapy ( $3 \mathrm{mg} / \mathrm{kg}$ ) cohort and the nivolumab $(3 \mathrm{mg} / \mathrm{kg})$ plus ipilimumab $(1 \mathrm{mg} / \mathrm{kg})$ cohort. Two treatmentrelated deaths were reported in the nivolumab $(1 \mathrm{mg} / \mathrm{kg})$ plus ipilimumab (3 $\mathrm{mg} / \mathrm{kg}$ ) cohort (including myasthenia gravis and worsening of renal failure), and one treatment-related pneumonitis caused death in the nivolumab $(3 \mathrm{mg} / \mathrm{kg})$ plus ipilimumab (1 mg/kg) cohort. The 1-year survival rate of patients with high TMBs was significantly higher (59b).

In a phase I dose-exploration and expansion study (NCT02261220), another double check point inhibitor group, durvalumab in combination with tremelimumab, demonstrated a promising clinical activity for ES-SCLC patients who received prior systematic therapy. The confirmed ORR was $13.3 \%$, and the median DOR was 18.9 months (95\% CI: 16.3-18.9). The disease control rate at 16 weeks was $20.0 \%$ (95\% CI: 7.7-38.6). The median PFS and median OS were 1.8 months and 7.9 months, respectively. The 12 -month OS was $41.7 \%$ (95\% CI: 23.3-59.2). However, $67 \%$ of patients experienced TRAEs, with $23 \%$ experiencing grade $3 / 4$ TRAEs. Fatigue (23\%) and pruritus (23\%) were the most common TRAEs (64).

In arm A of the BALTIC (NCT02937818) phase II study, the efficacy of durvalumab plus tremelimumab in platinumrefractory/resistant ES-SCLC patients was further tested. In this study, 25 patients were treated with durvalumab 
TABLE 2 | Clinical trials' efficacy data of ICls in patients with SCLC.

\begin{tabular}{|c|c|c|c|c|c|c|c|c|c|}
\hline Agent & Trial & Phase & $\begin{array}{l}\text { Line of } \\
\text { therapy }\end{array}$ & Population & Treatment arms & $\begin{array}{l}\text { Primary end } \\
\text { point }\end{array}$ & Secondary end points & $\begin{array}{l}\text { Median } \\
\text { follow-up time }\end{array}$ & $\begin{array}{l}\text { Publish } \\
\text { year }\end{array}$ \\
\hline \multirow[t]{2}{*}{ Nivolumab } & $\begin{array}{l}\text { CheckMate032 } \\
\text { (NCT01928394) }\end{array}$ & Phase 1/II & $\begin{array}{l}\text { Third or } \\
\text { later line }\end{array}$ & SCLC & $\begin{array}{l}\text { Nlvolumab } 3 \\
\mathrm{mg} / \mathrm{kg}\end{array}$ & $\begin{array}{l}\text { ORR: } 11.9 \%(95 \% \\
\text { Cl: } 6.5-19.5)\end{array}$ & $\begin{array}{l}\text { mDOR: } 17.9 \text { m (95\% } \\
\text { Cl:3.0-42.1); mOS: } 5.6 \mathrm{~m} \\
\text { (95\% Cl: 3.1-6.8); mPFS: } \\
1.4 \text { m (95\% Cl: } 1.3-1.6)\end{array}$ & $28.3 \mathrm{~m}$ & 2018 \\
\hline & $\begin{array}{l}\text { CheckMate331 } \\
\text { (NCT02481830) }\end{array}$ & Phaseiii & $\begin{array}{l}\text { Second } \\
\text { line }\end{array}$ & $\begin{array}{l}\text { Relapsed } \\
\text { SCLC }\end{array}$ & Nivolumab $240 \mathrm{mg}$ & $\begin{array}{l}\text { mOS: } 7.46 \mathrm{~m} \\
\text { (95\% Cl: } \\
5.65-9.20)\end{array}$ & $\begin{array}{l}\text { mPFS: } 1.45 \text { m (95\% } \\
\text { Cl:1.41- 1.51); ORR: } 13.7 \% \\
\text { (95\% Cl:10.0-18.3); } \\
\text { DOR:72\% }\end{array}$ & $15.8 \mathrm{~m}$ & 2018 \\
\hline \multirow[t]{9}{*}{ Pembrolizumab } & $\begin{array}{l}\text { KEYNOTE028 } \\
\text { (NCT02054806) }\end{array}$ & Phase lb & Third line & $\begin{array}{l}\text { ES- } \\
\text { SCLC }\end{array}$ & $\begin{array}{l}\text { Pembrolizumab } 10 \\
\mathrm{mg} / \mathrm{kg}\end{array}$ & $\begin{array}{l}\text { ORR: 33\% (95 Cl: } \\
16-55 \%)\end{array}$ & $\begin{array}{l}\text { mDOR: } 19.4 \text { m (95\% } \\
\text { Cl:3.6-20.0); mPFS:1.9m } \\
\text { (95\% Cl:1.7-5.9); mOS: } \\
\text { 9.7 m (95\% Cl: 4.1- not } \\
\text { reached). }\end{array}$ & $9.8 \mathrm{~m}$ & 2017 \\
\hline & $\begin{array}{l}\text { KEYNOTE158 } \\
\text { (NCT02628067) }\end{array}$ & Phase II & Third line & $\begin{array}{l}\text { ES- } \\
\text { SCLC }\end{array}$ & $\begin{array}{l}\text { Pembrolizumab } \\
200 \mathrm{mg}\end{array}$ & $\begin{array}{l}\text { ORR: } 18.7 \%(95 \% \\
\text { Cl: } 11.8 \% \\
-27.4 \%)\end{array}$ & $\begin{array}{l}\text { mPFS: } 2.0 \text { m (95\%Cl: } \\
\text { 1.9-2.1); mOS: } 8.7 \text { m (95\% } \\
\text { Cl: 5.6-12) }\end{array}$ & $10.1 \mathrm{~m}$ & 2018 \\
\hline & $\begin{array}{l}\text { pool analysis of } \\
\text { KEYNOTEO28 and } \\
\text { KEYNOTE158 }\end{array}$ & $\begin{array}{l}\text { Phase } \\
\text { Ib/phase } \\
\|\end{array}$ & Third line & $\begin{array}{l}\text { ES- } \\
\text { SCLC }\end{array}$ & $\begin{array}{l}\text { Pembrolizumab } 10 \\
\mathrm{mg} / \mathrm{kg} \text { or } 200 \mathrm{mg}\end{array}$ & $\begin{array}{l}\text { ORR: } 19.3 \%(95 \% \\
\text { Cl: } 11.4-29.4 \%)\end{array}$ & $\begin{array}{l}\text { mPFS: } 2.0 \text { m (95\% Cl: } \\
\text { 1.9-3.4); mOS:7.7 m (95\% } \\
\text { Cl: 5.2-10.1) }\end{array}$ & $25.9 m$ & 2020 \\
\hline & Gadgeel et al. (48) & Phase II & $\begin{array}{l}\text { Maintenance } \\
\text { therapy }\end{array}$ & $\begin{array}{l}\text { ES- } \\
\text { SCLC }\end{array}$ & $\begin{array}{l}\text { Pembrolizumab } \\
200 \mathrm{mg}\end{array}$ & $\begin{array}{l}\text { mPFS: } 1.4 \mathrm{~m}(95 \% \\
\text { Cl: } 1.3-2.8)\end{array}$ & $\begin{array}{l}\text { mOS: } 9.6 \mathrm{~m}(95 \% \mathrm{Cl}: \\
7.0-12)\end{array}$ & $5 w$ & 2018 \\
\hline & NCT02402920 & Phase I & $\begin{array}{l}\text { Second } \\
\text { line }\end{array}$ & $\begin{array}{l}\text { ES- } \\
\text { SCLC }\end{array}$ & $\begin{array}{l}45 \text { Gy thoracic } \\
\text { radiotherapy } \\
\text { +pembrolizumab } \\
50-200 \mathrm{mg}\end{array}$ & Safety & $\begin{array}{l}\text { mPFS: } 6.1 \mathrm{~m}(95 \% \mathrm{Cl} \\
\text { 4.1-8); mOS: } 8.4 \mathrm{~m} \mathrm{(95 \% ;} \\
\text { Cl: 6.7-10.1) }\end{array}$ & $7.3 \mathrm{~m}$ & 2020 \\
\hline & Welsh et al. (49) & phase I/II & - & $\begin{array}{l}\text { LS- } \\
\text { SCLC }\end{array}$ & $\begin{array}{l}\text { Concurrent } \\
\text { chemoradiotherapy } \\
\text { +pembrolizumab } \\
\text { 100-200 mg }\end{array}$ & Safety & $\begin{array}{l}\text { mPFS: } 19.7 \mathrm{~m} \text { (95\% Cl } \\
\text { 8.8-30.5); mOS:39.5 } \\
\text { months (95\% Cl:8.0-71.0) }\end{array}$ & $23.1 \mathrm{~m}$ & 2020 \\
\hline & NCT02551432 & Phase II & $\begin{array}{l}\text { Second } \\
\text { line }\end{array}$ & $\begin{array}{l}\text { ES- } \\
\text { SCLC }\end{array}$ & $\begin{array}{l}\text { Paclitaxel } \\
\text { +pembrolizumab } \\
200 \mathrm{mg}\end{array}$ & $\begin{array}{l}\text { ORR: } 23.1 \%(95 \% \\
\text { Cl: } 6.9-39.3)\end{array}$ & $\begin{array}{l}\text { mPFS: } 5.0 \text { m (95\% Cl: } \\
\text { 2.7-6.7); mOS:9.1m (95\% } \\
\text { Cl: 6.5-15.0) }\end{array}$ & $11.1 \mathrm{~m}$ & 2019 \\
\hline & $\begin{array}{l}\text { KEYNOTE604 } \\
\text { (NCT03066778) }\end{array}$ & Phase III & First line & $\begin{array}{l}\text { ES- } \\
\text { SCLC }\end{array}$ & $\begin{array}{l}\text { Pembrolizumab } \\
200 \mathrm{mg}+ \\
\text { etoposide+platinum }\end{array}$ & $\begin{array}{l}\text { mPFS: } 4.5 \text { m (95\% } \\
\text { Cl: } 4.3-5.4) ; \text { mOS: } \\
\text { 10.8 m (95\% Cl: } \\
\text { 9.2-12.9) }\end{array}$ & $\begin{array}{l}\text { ORR: } 70.6 \% \text { (95\% Cl: } \\
\text { 64.2-76.4); mDOR: 4.2 m } \\
\text { (95\% Cl:1.01-26.01) }\end{array}$ & $22 m$ & 2020 \\
\hline & & & & & $\begin{array}{l}\text { Placebo + } \\
\text { etoposide } \\
\text { +platinum }\end{array}$ & $\begin{array}{l}\text { mPFS: } 4.3 \mathrm{~m} \text { (95\% } \\
\text { Cl: } 4.2-4.4) ; \text { mOS: } \\
9.7 \text { m (95\% Cl: } \\
\text { 8.6-10.7) }\end{array}$ & $\begin{array}{l}\text { ORR: } 61.8 \% \text { (95\% Cl: } \\
\text { 55.1-68.2); mDOR: } 3.7 \mathrm{~m} \\
\text { (95\% Cl:1.41-25.81) }\end{array}$ & & \\
\hline Tislelizumab & NCT03432598 & Phase II & First line & $\begin{array}{l}\text { ES- } \\
\text { SCLC }\end{array}$ & $\begin{array}{l}\text { Tislelizumab } \\
200 \mathrm{mg}+ \\
\text { etoposide+platinum }\end{array}$ & $\begin{array}{l}\text { ORR: } 77 \%(95 \% \\
\text { Cl: } 50.1-93.2)\end{array}$ & $\begin{array}{l}\text { mPFS: } 6.9 \text { m (95\% Cl: } \\
4.9-10.09)\end{array}$ & $15.3 \mathrm{~m}$ & 2020 \\
\hline
\end{tabular}


TABLE 2 | Continued

\begin{tabular}{|c|c|c|c|c|c|c|c|c|c|}
\hline Agent & Trial & Phase & $\begin{array}{l}\text { Line of } \\
\text { therapy }\end{array}$ & Population & Treatment arms & $\begin{array}{l}\text { Primary end } \\
\text { point }\end{array}$ & Secondary end points & $\begin{array}{l}\text { Median } \\
\text { follow-up time }\end{array}$ & $\begin{array}{l}\text { Publish } \\
\text { year }\end{array}$ \\
\hline \multirow[t]{5}{*}{ Atezolizumab } & NCT01375842 & Phase la & First line & $\begin{array}{l}\text { ES- } \\
\text { SCLC }\end{array}$ & $\begin{array}{l}\text { Atezolizumab } 15 \\
\mathrm{mg} / \mathrm{kg} \text { or } 1200 \mathrm{mg}\end{array}$ & Safety & $\begin{array}{l}\text { ORR: 6\%; mPFS: } 1.5 \mathrm{~m} \\
\text { (95\% Cl: } 1.2-2.7) ; \text { mOS: } \\
5.9 \text { m (95\% Cl: 4.3-20.1) }\end{array}$ & $6.7 \mathrm{~m}$ & 2016 \\
\hline & $\begin{array}{l}\text { IMpower133 } \\
\text { (NCT02763579) }\end{array}$ & $\begin{array}{l}\text { Phase } \\
\text { I/III }\end{array}$ & First line & $\begin{array}{l}\text { ES- } \\
\text { SCLC }\end{array}$ & $\begin{array}{l}\text { Atezolizumab } \\
1,200 \\
\text { mg+carboplatin }+ \\
\text { etoposide }\end{array}$ & $\begin{array}{l}\text { mOS: } 12.3 \mathrm{~m} \\
(95 \% \\
\text { Cl:10.8-15.9); } \\
\text { mPFS:5.2 m (95\% } \\
\text { Cl: 4.4-5.6) }\end{array}$ & $\begin{array}{l}\text { ORR: } 60.2 \% \text { (95\% } \\
\text { Cl:53.1-67.0); DOR: } 4.2 \mathrm{~m} \\
\text { (95\%Cl: 1.4+-19.5) }\end{array}$ & $13.9 m$ & 2018 \\
\hline & & & & & $\begin{array}{l}\text { Placebo+ } \\
\text { carboplatin+ } \\
\text { etoposide }\end{array}$ & $\begin{array}{l}\text { mOS: } 10.3 \mathrm{~m}(95 \% \\
\text { Cl: 9.3-11.3); } \\
\text { mPFS: 4.3m (95\% } \\
\text { Cl: 4.2-4.5) }\end{array}$ & $\begin{array}{l}\text { ORR: } 64.4 \% \text { (95\% Cl: } \\
\text { 57.3-71.0); DOR: } 3.9 \mathrm{~m} \\
\text { (95\% Cl:2.0-16.1+) }\end{array}$ & & \\
\hline & $\begin{array}{l}\text { IFCT-1603 } \\
\text { (NCT03059667) }\end{array}$ & Phase II & $\begin{array}{l}\text { Second } \\
\text { line }\end{array}$ & $\begin{array}{l}\text { relapsed } \\
\text { ES- } \\
\text { SCLC }\end{array}$ & $\begin{array}{l}\text { Atezolizumab } \\
1,200 \mathrm{mg}\end{array}$ & $\begin{array}{l}\text { ORR: } 2.3 \%(95 \% \\
\text { Cl: } 0.0-6.8)\end{array}$ & $\begin{array}{l}\text { mPFS:1.4 m (95\%Cl: } \\
\text { 1.2-1.5); mOS: } 9.5 \text { m (95\% } \\
\text { Cl: 3.2-14.4) }\end{array}$ & $13.7 \mathrm{~m}$ & 2019 \\
\hline & & & & & Chemotherapy & $\begin{array}{l}\text { ORR: } 10 \%(95 \% \\
\text { Cl: } 0.0-23.1)\end{array}$ & $\begin{array}{l}\text { mPFS: } 4.3 \mathrm{~m} \text { (95\%Cl: } \\
\text { 1.5-5.9); mOS:8.7m (95\% } \\
\text { Cl:4.1-12.7) }\end{array}$ & & \\
\hline \multirow[t]{3}{*}{ Durvalumab } & $\begin{array}{l}\text { CASPIAN } \\
\text { (NCT03043872) }\end{array}$ & Phase III & First line & $\begin{array}{l}\text { ES- } \\
\text { SCLC }\end{array}$ & $\begin{array}{l}\text { Durvalumab } \\
1,500 \mathrm{mg}+ \\
\text { etoposide+ } \\
\text { platinum }\end{array}$ & $\begin{array}{l}\text { mOS: } 13.0 \mathrm{~m} \\
\text { (95\% Cl: } \\
11.5-14.8)\end{array}$ & $\begin{array}{l}\text { mPFS: } 5.1 \mathrm{~m}(95 \% \mathrm{Cl} \\
4.7-6.2) ; \text { ORR: } 68 \%\end{array}$ & $14.2 \mathrm{~m}$ & 2019 \\
\hline & & & & & Etoposide+platinum & $\begin{array}{l}\text { mOS: } 10.3 \mathrm{~m} \\
\text { (95\%Cl: 9.3-11.2) }\end{array}$ & $\begin{array}{l}\text { mPFS: } 5.4 \text { m (95\% } \\
\text { Cl:4.8-6.2); ORR: 58\% }\end{array}$ & & \\
\hline & Goldman et al. (36) & Phase I/II & $\begin{array}{l}\text { Second } \\
\text { line }\end{array}$ & $\begin{array}{l}\text { Relapsed } \\
\text { SCLC }\end{array}$ & $\begin{array}{l}\text { Durvalumab } 10 \\
\mathrm{mg} / \mathrm{kg}\end{array}$ & Safety & $\begin{array}{l}\text { ORR: } 9.5 \% \text { (95\% Cl: } \\
\text { 1.2-30.4); mPFS: } 1.5 \mathrm{~m} \\
\text { (95\% Cl: 0.9-1.8); mOS: } \\
\text { 4.8m (95\% Cl: 1.3-10.4) }\end{array}$ & NA & 2018 \\
\hline \multirow[t]{4}{*}{ Ipilimumab } & $\begin{array}{l}\text { CA184-041 } \\
\text { (NCT00527735) }\end{array}$ & Phase II & First line & $\begin{array}{l}\text { ES- } \\
\text { SCLC }\end{array}$ & $\begin{array}{l}\text { Placebo/ paclitaxel } \\
\text { /carboplatin }\end{array}$ & irPFS: $5.3 \mathrm{~m}$ & $\begin{array}{l}\text { mOS: } 9.9 \text { m; irBORR: 53\% } \\
\text { (95\% Cl: 38-68\%); irDCR: } \\
\text { 96\% (95\% Cl: 85-100\%) }\end{array}$ & $11.1 \mathrm{~m}$ & 2013 \\
\hline & & & & & $\begin{array}{l}\text { Ipilimumab } 10 \\
\mathrm{mg} / \mathrm{kg} / \mathrm{placebo+} \\
\text { paclitaxel/ } \\
\text { carboplatin(concurrent) }\end{array}$ & irPFS: $5.7 \mathrm{~m}$ & $\begin{array}{l}\text { mOS: } 9.1 \text { m; irBORR: 49\% } \\
\text { (95\% Cl: 33-65\%); irDCR: } \\
\text { 81\% (95\% Cl: 67-92\%) }\end{array}$ & & \\
\hline & & & & & $\begin{array}{l}\text { Ipilimumab } 10 \\
\text { mg/kg/placebo+ } \\
\text { paclitaxel/ } \\
\text { carboplatin(phased) }\end{array}$ & irPFS: $6.4 \mathrm{~m}$ & $\begin{array}{l}\text { mOS: } 12.9 \text { m; irBORR: } 71 \% \\
\text { (95\% Cl: } 55 \%-84 \%) \text {; irDCR: } \\
\text { 93\% (95\% Cl: 81-99\%) }\end{array}$ & & \\
\hline & NCT01331525 & Phase II & First line & $\begin{array}{l}\text { ES- } \\
\text { SCLC }\end{array}$ & $\begin{array}{l}\text { Ipilimumab } 10 \\
\mathrm{mg} / \mathrm{kg}+ \\
\text { carboplatin+ } \\
\text { etoposide }\end{array}$ & not meet & $\begin{array}{l}\text { mPFS: } 6.9 \text { m (95\%Cl: } \\
\text { 5.5-7.9); mOS: } 17.0 \mathrm{~m} \\
\text { (95\% Cl: } 7.9-24.3) ; \text { median } \\
\text { irPFS:7.3 m (95\% Cl: } \\
\text { 5.5-8.8) }\end{array}$ & $8.5 \mathrm{~m}$ & 2016 \\
\hline
\end{tabular}


TABLE 2 | Continued

\begin{tabular}{|c|c|c|c|c|c|c|c|c|c|}
\hline Agent & Trial & Phase & $\begin{array}{l}\text { Line of } \\
\text { therapy }\end{array}$ & Population & Treatment arms & $\begin{array}{l}\text { Primary end } \\
\text { point }\end{array}$ & Secondary end points & $\begin{array}{l}\text { Median } \\
\text { follow-up time }\end{array}$ & $\begin{array}{l}\text { Publish } \\
\text { year }\end{array}$ \\
\hline & NCT01450761 & Phase III & First line & $\begin{array}{l}\text { ES- } \\
\text { SCLC }\end{array}$ & $\begin{array}{l}\text { Ipilimumab } 10 \\
\text { mg/kg+etoposide } \\
\text { +platinum } \\
\text { (cisplatin+ } \\
\text { carboplatin) }\end{array}$ & mOS: $11.0 \mathrm{~m}$ & $\begin{array}{l}\text { mPFS: } 4.6 \text { m; mDOR: } 4.01 \\
\text { (95\% Cl: 3.32-4.17) }\end{array}$ & $10.5 \mathrm{~m}$ & 2016 \\
\hline & & & & & $\begin{array}{l}\text { Placebo+ } \\
\text { etoposide+ } \\
\text { platinum } \\
\text { (cisplatin+ } \\
\text { carboplatin) }\end{array}$ & mOS: $10.9 \mathrm{~m}$ & $\begin{array}{l}\text { mPFS: } 4.4 \text { m; mDOR: } \\
3.45 \text { m (95\% Cl: 3.25-4.07) }\end{array}$ & $10.2 \mathrm{~m}$ & \\
\hline \multirow[t]{6}{*}{$\begin{array}{l}\text { Nivolumab } \\
\text { +ipilimumab }\end{array}$} & $\begin{array}{l}\text { CheckMate451 } \\
\text { (NCT02538666) }\end{array}$ & Phase III & $\begin{array}{l}\text { Maintenance } \\
\text { therapy }\end{array}$ & $\begin{array}{l}\text { Relapsed } \\
\text { ES- } \\
\text { SCLC }\end{array}$ & $\begin{array}{l}\text { Nivolumab } 1 \\
\text { mg/kg + } \\
\text { ipilimumab } 3 \\
\text { mg/kg }\end{array}$ & $\begin{array}{l}\text { mOS: } 9.17 \mathrm{~m} \\
(95 \% \\
\text { Cl:8.15-10.25) }\end{array}$ & $\begin{array}{l}\text { mPFS: } 1.74(95 \% \mathrm{Cl}: \\
1.48-2.63)\end{array}$ & $9 m$ & 2019 \\
\hline & & & & & $\begin{array}{l}\text { Nivolumab } 1 \\
\mathrm{mg} / \mathrm{kg}\end{array}$ & $\begin{array}{l}\text { mOS: } 10.41 \mathrm{~m} \\
(95 \% \\
\text { Cl:9.46-12.12) }\end{array}$ & $\begin{array}{l}\text { mPFS: } 1.87 \text { (95\% Cl: } \\
1.61-2.63)\end{array}$ & & \\
\hline & & & & & Placebo & $\begin{array}{l}\text { mOS: } 9.56 \mathrm{~m} \\
(95 \% \\
\text { Cl:8.18-11.01) }\end{array}$ & $\begin{array}{l}\text { mPFS: } 1.45 \text { (95\% Cl: } \\
1.41-1.48)\end{array}$ & & \\
\hline & $\begin{array}{l}\text { CheckMate032 } \\
\text { (NCT01928394) }\end{array}$ & Phase I/II & $\begin{array}{l}\text { Second } \\
\text { or later } \\
\text { line }\end{array}$ & SCLC & $\begin{array}{l}\text { Nivolumab } \\
3 \mathrm{mg} / \mathrm{kg}\end{array}$ & ORR:10\% & $\begin{array}{l}\text { mOS: } 4.4 \mathrm{~m} \text { (95\% Cl: } \\
\text { 3.0-9.3); mPFS: } 1.4 \text { m (95\% } \\
\text { Cl: } 1.4-1.9)\end{array}$ & $198.5 d$ & 2016 \\
\hline & & & & & $\begin{array}{l}\text { Nivolumab } 1 \\
\text { mg/kg + } \\
\text { ipilimumab } 3 \\
\text { mg/kg }\end{array}$ & ORR:23\% & $\begin{array}{l}\text { mOS: } 7.7 \text { m (95\% Cl: } \\
\text { 3.6-18.0); mPFS: } 2.6 \mathrm{~m} \\
(95 \% \text { Cl: } 1.4-4.1)\end{array}$ & $361.0 \mathrm{~d}$ & \\
\hline & & & & & $\begin{array}{l}\text { Nivolumab } 3 \\
\text { mg/kg }+ \\
\text { ipilimumab } 1 \\
\text { mg/kg }\end{array}$ & ORR:19\% & $\begin{array}{l}\text { mOS: } 6.0 \mathrm{~m} \text { (95\% Cl: } \\
\text { 3.6-11.0); mPFS: } 1.4 \mathrm{~m} \\
(95 \% \mathrm{Cl}: 1.3-2.2)\end{array}$ & $260.5 d$ & \\
\hline \multirow[t]{2}{*}{$\begin{array}{l}\text { Durvalumab+ } \\
\text { tremelimumab }\end{array}$} & NCT02261220 & Phase I & Third line & $\begin{array}{l}\text { ES- } \\
\text { SCLC }\end{array}$ & $\begin{array}{l}\text { Durvalumab } 20 \\
\mathrm{mg} / \mathrm{kg}+\text { tremelimumab } \\
1 \mathrm{mg} / \mathrm{kg}\end{array}$ & safety & $\begin{array}{l}\text { ORR: 13.3\%; DOR: } 18.9 \mathrm{~m} \\
\text { (95\% Cl: 16.3-18.9); mPFS: } \\
\text { 1.8 m (95\% Cl: 1.0-1.9); } \\
\text { mOS: 7.9 m (95\% Cl: } \\
\text { 3.2-15.8) }\end{array}$ & $N R$ & 2018 \\
\hline & $\begin{array}{l}\text { BALTIC } \\
\text { (NCT02937818) }\end{array}$ & Phase II & First line & $\begin{array}{l}\text { ES- } \\
\text { SCLC }\end{array}$ & $\begin{array}{l}\text { Durvalumab } \\
1,500 \mathrm{mg}+ \\
\text { tremelimumab } \\
75 \mathrm{mg}\end{array}$ & $\begin{array}{l}\text { ORR: } 9.5 \%(95 \% \\
\text { Cl: } 1.17-30.38)\end{array}$ & 12 weeks DCR: $38.1 \%$ & $14 w$ & 2018 \\
\hline
\end{tabular}




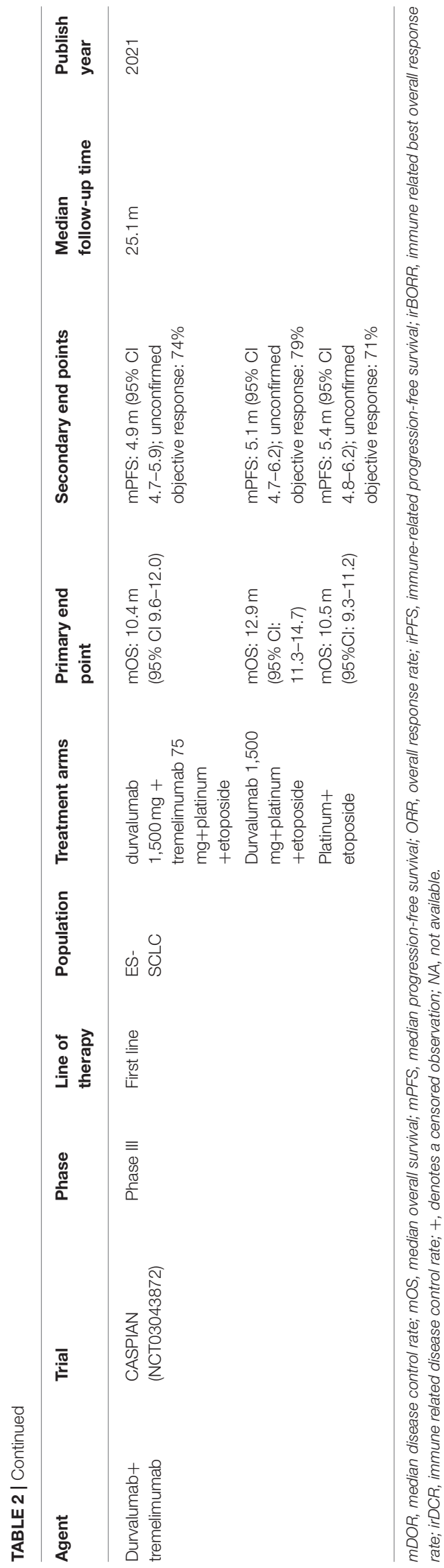

$(1,500 \mathrm{mg})$ plus tremelimumab $(75 \mathrm{mg})$ for up to 4 months, followed by durvalumab $(1,500 \mathrm{mg})$ until progressive disease or discontinuation. The reported ORR was 9.5\% (95\% CI: $1.17-30.38$ ); $23.8 \%$ of patients had stable disease and $4.8 \%$ of patients had an unconfirmed partial response. Grade 3 or higher TRAEs were experienced by $19 \%$ of patients; however, the updated information has not yet been published (65).

On December 2020, the updated results of CASPIAN trial published the data of durvalumab plus chemotherapy group and durvalumab plus tremelimumab plus chemotherapy group. Safety profiles of the durvalumab plus chemotherapy group and the chemotherapy group were consistent with previously reported. Immune-mediated adverse events were reported in patients in the durvalumab plus tremelimumab plus chemotherapy group, durvalumab plus chemotherapy group and chemotherapy group were 36,20 , and $3 \%$, respectively. Usually reported irAEs were hypothyroid events, hyperthyroid events, diarrhea or colitis and dermatitis or rash. Grade 3 or 4 immune mediated adverse events occurred in $14 \%$ patients in the durvalumab plus tremelimumab plus chemotherapy group, $5 \%$ patients in the durvalumab plus chemotherapy group, and $<1 \%$ patients in chemotherapy group. Deaths caused by irAEs occurred in $1 \%$ patients receiving durvalumab plus tremelimumab plus chemotherapy (enterocolitis, pneumonitis, pneumonitis and hepatitis), $1 \%$ patients receiving durvalumab plus platinum-etoposide (hepatotoxicity and interstitial lung disease) and $<1 \%$ receiving platinum-etoposide (pneumonitis) (66).

\section{DISCUSSION}

For many years, few breakthroughs in SCLCs have been reported. Chemotherapy and radiotherapy were the only effective therapeutic methods for ES-SCLC patients. However, in recent years, immunotherapy has brought new hope for patients with SCLC. Some ICIs have improved chemotherapy's efficacy in ES-SCLC patients, but a comprehensive understanding of the mechanisms and preclinical rationale of immunotherapy in SCLC patients is still required. In this review, we summarized the available clinical trial data on ICIs for the treatment of SCLC. We are particularly concerned about IRAES, which are often overlooked by existing reviews.

A systematic collection of the efficacy and safety data of ICIs in the treatment of SCLC is performed in this review. Two reviewers independently searched current literature from the Cochrane Library, Clinical Trials, PubMed, and MEDLINE databases, using the following key words: "Small cell lung cancer," "immune checkpoint inhibitor," "nivolumab," "pembrolizumab," "atezolizumab," "avelumab," "durvalumab," and "ipilimumab." Clinical trials reporting both efficacy and safety data were included. A total of 23 studies covering 5 PD-1/PD-L1 inhibitors and 1 CTLA-4 antibody were included.

To intuitively compare the efficacy of ICIs and the occurrence of irAEs in SCLC, we summarized the results in Tables 2, 3. Overall, the efficacy of different mechanisms in ICIs also varied (Table 2). The anti-PD1 inhibitors nivolumab and 
TABLE 3 | Clinical trials' safety data of ICls in patients with SCLC.

\begin{tabular}{|c|c|c|c|c|c|c|c|c|c|c|}
\hline Target & NCT number & Treatment & $\begin{array}{l}\text { Enrolled } \\
\text { number }\end{array}$ & TRAEs & $\begin{array}{l}\text { TRAEs } \\
\text { (grade } \geq 3 \text { ) }\end{array}$ & IRAEs & $\begin{array}{c}\text { IRAEs } \\
\text { (grade } \geq 3 \text { ) }\end{array}$ & Most common TRAEs/IRAEs & TRAEs/IRAEs (grade $\geq 3$ ) & $\begin{array}{l}\text { Death related to } \\
\text { TRAEs/IRAEs }\end{array}$ \\
\hline \multirow[t]{7}{*}{ PD-1 } & $\begin{array}{l}\text { CheckMate032 } \\
\text { (NCT01928394) }\end{array}$ & $\begin{array}{l}\text { Nivolumab } \\
3 \mathrm{mg} / \mathrm{kg}\end{array}$ & 109 & $55 \%$ & $11.9 \%$ & $48 \%$ & $4 \%$ & $\begin{array}{l}\text { - IRAEs: } \\
\text { - Skin reactions }(21.1 \%), \\
\text { - Endocrine }(9.2 \%), \\
\text { - Gastrointestinal }(6.4 \%), \\
\text { - Hepatic (4.6\%), } \\
\text { - Infusion reaction }(3.7 \%), \\
\text { - } \text { Pulmonary (1.8\%), renal } \\
\\
(0.9 \%)\end{array}$ & $\begin{array}{l}\text { - IRAEs: } \\
\text { - Pneumonitis (1.8\%), } \\
\text { - Rash }(0.9 \%) \text {, } \\
\text { - } \text { AST increased }(0.9 \%)\end{array}$ & Pneumonitis (0.9\%) \\
\hline & CheckMate331 & Nivolumab $240 \mathrm{mg}$ & 282 & $55 \%$ & $14 \%$ & NA & NA & NA & NA & NA \\
\hline & & $\begin{array}{l}\text { Chemotherapy } \\
\text { (either topotecan } \\
\text { or amrubicin) }\end{array}$ & 265 & $90 \%$ & $73 \%$ & NA & NA & NA & NA & NA \\
\hline & $\begin{array}{l}\text { KEYNOTE028 } \\
\text { (NCT02054806) }\end{array}$ & $\begin{array}{l}\text { Pembrolizumab } \\
10 \mathrm{mg} / \mathrm{kg}\end{array}$ & 24 & $66.7 \%$ & $8.3 \%$ & NA & NA & $\begin{array}{l}\text { - } \text { TRAEs: } \\
\text { - Arthralgia (16.6\%), } \\
\text { - } \text { Asthenia (16.6\%), } \\
\text { - } \text { Rash (16.7\%), } \\
\text { - } \text { Diarrhea (12.5\%), fatigue } \\
\quad(12.5 \%)\end{array}$ & $\begin{array}{l}\text { - TRAEs: } \\
\text { - Grade } 3 \text { bilirubin } \\
\text { elevation (4.2\%), } \\
\text { - Grade } 5 \text { colitis/intestinal } \\
\text { ischemia }(4.2 \%)\end{array}$ & $\begin{array}{l}\text { Colitis and intestinal } \\
\text { ischemia }(4.2 \%)\end{array}$ \\
\hline & $\begin{array}{l}\text { KEYNOTE158 } \\
\text { (NCT02628067) }\end{array}$ & $\begin{array}{l}\text { Pembrolizumab } \\
200 \mathrm{mg}\end{array}$ & 107 & $60 \%$ & $12 \%$ & $33 \%$ & $5 \%$ & $\begin{array}{l}\text { - IRAEs: } \\
\text { - Hypothyroidism (12\%), } \\
\text { - Hyperthyroidism (7\%), } \\
\text { - } \text { Severe skin reactions }(3 \%), \\
\text { - } \text { Adrenal insufficiency }(2 \%), \\
\text { nephritis }(2 \%), \\
\text { - } \text { Pneumonitis }(2 \%), \text { pancreatitis } \\
\text { (2\%) }\end{array}$ & $\begin{array}{l}\text { - IRAEs: } \\
\text { - Severe skin reactions } \\
\text { ( }(1 \%), \\
\text { - Adrenal insufficiency } \\
\text { ( }(1 \%), \\
\text { - Pancreatitis }(2 \%), \\
\text { - Pneumonitis }(1 \%) \text {, colitis } \\
\text { (1\%) }\end{array}$ & $\begin{array}{l}\text { - Pneumonitis (0.9\%), } \\
\text { - Encephalopathy (0.9\%) }\end{array}$ \\
\hline & $\begin{array}{l}\text { Pool analysis of } \\
\text { KEYNOTE-028 } \\
\text { and } \\
\text { KEYNOTE-158 }\end{array}$ & $\begin{array}{l}\text { Pembrolizumab } \\
10 \mathrm{mg} / \mathrm{kg} \text { or } \\
200 \mathrm{mg}\end{array}$ & 83 & $61.4 \%$ & $7.2 \%$ & $24.1 \%$ & $6 \%$ & 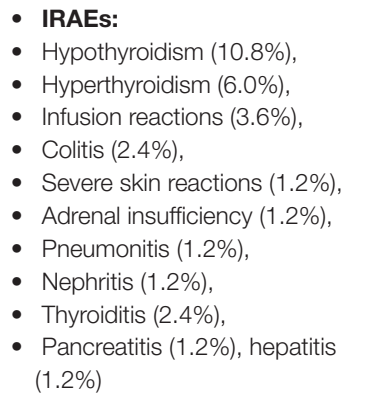 & $\begin{array}{l}\text { - IRAEs: } \\
\text { - } \text { Colitis (2.4\%), } \\
\text { - Adrenal insufficiency } \\
\text { (1.2\%), } \\
\text { - Pancreatitis }(1.2 \%), \\
\text { - Pneumonitis (1.2\%) }\end{array}$ & $\begin{array}{l}\text { - Pneumonia (1.2\%), } \\
\text { - Intestinal ischemia (1.2\%), } \\
\text { - Encephalopathy (1.2\%) }\end{array}$ \\
\hline & Gadgeel et al. (48) & $\begin{array}{l}\text { Pembrolizumab } \\
200 \mathrm{mg}\end{array}$ & 45 & NA & NA & NA & NA & $\begin{array}{l}\text { - IRAEs: } \\
\text { - Rash ( }(18 \%), \\
\text { - Hypothyroidism ( } 9 \%), \\
\text { - Type I diabetes mellitus with } \\
\text { diabetic Ketoacidosis (11\%) }\end{array}$ & None & None \\
\hline
\end{tabular}


TABLE 3 | Continued

\begin{tabular}{|c|c|c|c|c|c|c|c|c|c|c|}
\hline Target & NCT number & Treatment & $\begin{array}{l}\text { Enrolled } \\
\text { number }\end{array}$ & TRAEs & $\begin{array}{c}\text { TRAEs } \\
\text { (grade } \geq 3 \text { ) }\end{array}$ & IRAEs & $\begin{array}{c}\text { IRAEs } \\
\text { (grade } \geq 3 \text { ) }\end{array}$ & Most common TRAEs/IRAEs & TRAEs/IRAEs (grade $\geq 3$ ) & $\begin{array}{l}\text { Death related to } \\
\text { TRAEs/IRAEs }\end{array}$ \\
\hline & NCT02402920 & $\begin{array}{l}45 \text { Gy thoracic } \\
\text { radiotherapy + } \\
\text { pembrolizumab } \\
50-200 \mathrm{mg}\end{array}$ & 33 & NA & NA & NA & NA & NA & NA & NA \\
\hline & Welsh et al. (49) & $\begin{array}{l}\text { Concurrent } \\
\text { chemoradiotherapy } \\
\text { + pembrolizumab } \\
\text { 100-200 mg }\end{array}$ & 40 & $100 \%$ & $88 \%$ & NA & NA & $\begin{array}{l}\text { - TRAEs: } \\
\text { - Fatigue }(60 \%), \\
\text { - Dysphagia (58\%), } \\
\text { - Dyspnea }(50 \%), \\
\text { - Esophagitis }(43 \%), \\
\text { - Nausea (35\%) }\end{array}$ & $\begin{array}{l}\text { - } \quad \text { TRAEs: } \\
\text { - } \text { Anemia (13\%), } \\
\text { - } \quad \text { Neutropenia (13\%), } \\
\text { - Lung infection (8\%), } \\
\text { - } \text { Pneumonitis }(8 \%)\end{array}$ & None \\
\hline & NCT02551432 & $\begin{array}{l}\text { Paclitaxel + } \\
\text { pembrolizumab } \\
200 \text { mg }\end{array}$ & 26 & $100 \%$ & $46 \%$ & NA & NA & $\begin{array}{l}\text { - } \text { TRAEs: } \\
\text { - } \text { Peripheral sensory } \\
\text { neuropathy }(57.7 \%), \\
\text { - } \text { Myalgia (34.6\%), } \\
\text { - } \text { Anemia }(23.1 \%), \\
\text { - } \text { Diarrhea (23.1\%), } \\
\text { - } \text { Anorexia }(19.2 \%), \\
\text { - } \text { Pneumonia (19.2\%) }\end{array}$ & $\begin{array}{l}\text { - TRAEs: } \\
\text { - Neutropenia }(7.7 \%), \\
\text { - Febrile neutropenia } \\
\text { ( } 7.7 \%), \\
\text { - Asthenia }(7.7 \%), \\
\text { - Hyponatremia }(7.7 \%), \\
\text { - Type I diabetes mellitus } \\
\text { - }(3.9 \%), \\
\text { - Anemia }(3.9 \%), \\
\text { - Myalgia }(3.9 \%)\end{array}$ & None \\
\hline & \multirow[t]{2}{*}{$\begin{array}{l}\text { KEYNOTE-604 } \\
\text { (NCT03066778) }\end{array}$} & $\begin{array}{l}\text { Pembrolizumab } \\
200 \mathrm{mg}+ \\
\text { etoposide }+ \\
\text { platinum }\end{array}$ & 223 & $97.8 \%$ & $63.7 \%$ & $24.7 \%$ & $8.1 \%$ & $\begin{array}{l}\text { - IRAEs: } \\
\text { - Hypothyroidism (10.3\%), } \\
\text { - Hyperthyroidism }(6.7 \%), \\
\text { - } \text { Pneumonitis (4.0\%), } \\
\text { - Severe skin reactions (2.2\%), } \\
\text { - Hepatitis }(1.8 \%), \\
\text { - } \text { Colitis }(1.3 \%), \\
\text { - } \text { Adrenal insufficiency }(0.9 \%), \\
\text { - Hypophysitis }(0.9 \%), \\
\text { - } \text { Nephritis }(0.9 \%), \\
\text { - Encephalitis }(0.9 \%), \\
\text { - } \text { Myositis }(0.4 \%), \\
\text { - Pancreatitis }(0.4 \%), \\
\text { - Type } 1 \text { diabetes mellitus } \\
\text { - }(0.4 \%), \\
\text { - Uveitis }(0.4 \%)\end{array}$ & $\begin{aligned} \text { - } & \text { IRAEs: } \\
\text { - } & \text { Severe skin reactions } \\
& (1.8 \%), \text { pneumonitis } \\
& (1.3 \%), \\
\text { - } & \text { Hepatitis }(1.3 \%), \\
\text { - } & \text { Adrenal insufficiency } \\
& (0.9 \%), \\
\text { - } & \text { Hyperthyroidism }(0.4 \%), \\
\text { - } & \text { Colitis }(0.4 \%), \\
\text { - } & \text { Nephritis }(0.4 \%), \\
\text { - } & \text { Myositis }(0.4 \%), \\
\text { - } & \text { Pancreatitis }(0.4 \%), \\
\text { - } & \text { Type } 1 \text { diabetes mellitus } \\
& (0.4 \%), \text { uveitis }(0.4 \%)\end{aligned}$ & $\begin{array}{l}\text { - Neutropenic sepsis } \\
\text { ( } 1.3 \%), \\
\text { - Cardiopulmonary failure } \\
(0.4 \%), \\
\text { - Respiratory failure }(0.4 \%) \text {, } \\
\text { - Sepsis }(0.4 \%)\end{array}$ \\
\hline & & $\begin{array}{l}\text { Placebo + } \\
\text { etoposide } \\
\text { platinum }\end{array}$ & 223 & $95.5 \%$ & $61 \%$ & $10.3 \%$ & $0.9 \%$ & $\begin{array}{l}\text { - IRAEs: } \\
\text { - Hypothyroidism (2.2\%), } \\
\text { - Hyperthyroidism (2.7\%), } \\
\text { - Pneumonitis (2.2\%), } \\
\text { - Severe skin reactions }(0.9 \%), \\
\text { - } \text { Colitis }(0.9 \%), \\
\text { - } \text { Adrenal insufficiency }(0.4 \%), \\
\text { - } \text { Myasthenic syndrome }(0.4 \%), \\
\text { - } \text { Myocarditis }(0.4 \%)\end{array}$ & $\begin{array}{l}\text { - IRAEs: } \\
\text { - Colitis (0.9\%) }\end{array}$ & Neutropenic sepsis $(0.4 \%)$ \\
\hline
\end{tabular}


TABLE 3 | Continued

\begin{tabular}{|c|c|c|c|c|c|c|c|c|c|c|}
\hline Target & NCT number & Treatment & $\begin{array}{l}\text { Enrolled } \\
\text { number }\end{array}$ & TRAEs & $\begin{array}{l}\text { TRAEs } \\
\text { (grade } \geq 3 \text { ) }\end{array}$ & IRAEs & $\begin{array}{c}\text { IRAEs } \\
\text { (grade } \geq 3 \text { ) }\end{array}$ & Most common TRAEs/IRAEs & TRAEs/IRAEs (grade $\geq 3$ ) & $\begin{array}{l}\text { Death related to } \\
\text { TRAEs/IRAEs }\end{array}$ \\
\hline & NCT03432598 & $\begin{array}{l}\text { Tislelizumab } \\
200 \mathrm{mg}+ \\
\text { etoposide }+ \\
\text { platinum }\end{array}$ & 17 & $100 \%$ & $76.5 \%$ & $35.3 \%$ & None & $\begin{array}{l}\text { - IRAEs: } \\
\text { - Thyroid disorders (29.4\%), } \\
\text { - Pneumonitis (5.9\%), } \\
\text { - Type } 1 \text { diabetes mellitus } \\
(5.9 \%)\end{array}$ & None & None \\
\hline \multirow[t]{5}{*}{ PD-L1 } & NCT01375842 & $\begin{array}{l}\text { Atezolizumab } \\
15 \mathrm{mg} / \mathrm{kg} \text { or } \\
1,200 \mathrm{mg}\end{array}$ & 17 & $65 \%$ & $17.6 \%$ & NA & NA & $\begin{array}{l}\text { - TRAEs: } \\
\text { - Fatigue (24\%) }\end{array}$ & $\begin{array}{l}\text { - TRAEs: } \\
\text { - Pneumonitis }(5.9 \%) \text {, } \\
\text { - Hepatic failure }(5.9 \%)\end{array}$ & None \\
\hline & $\begin{array}{l}\text { IMpower133 } \\
\text { (NCT02763579) }\end{array}$ & $\begin{array}{l}\text { Atezolizumab } \\
1,200 \mathrm{mg}+ \\
\text { carboplatin }+ \\
\text { etoposide }\end{array}$ & 198 & $94.9 \%$ & $58.1 \%$ & $39.9 \%$ & $10.5 \%$ & 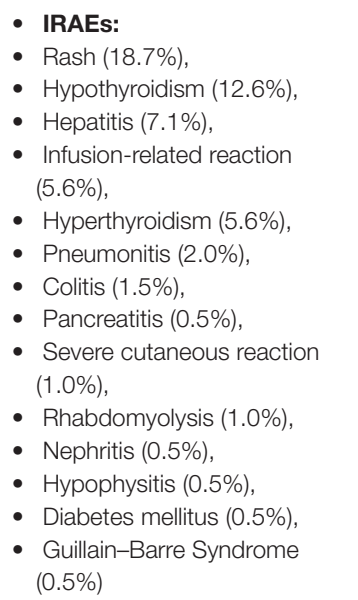 & $\begin{array}{l}\text { - IRAEs: } \\
\text { - Rash (2.0\%), } \\
\text { - Hepatitis (1.5\%), } \\
\text { - Infusion-related reaction } \\
\text { ( } 2.0 \%), \\
\text { - Pneumonitis (0.5\%), } \\
\text { - } \text { Colitis ( } 1.0 \%), \\
\text { - Pancreatitis (0.5\%), } \\
\text { - Rhabdomyolysis (0.5\%), } \\
\text { - } \text { Nephritis (0.5\%), } \\
\text { ( }(0.5 \%)\end{array}$ & $\begin{array}{l}\text { - } \quad \text { Neutropenia }(0.5 \%), \\
\text { - Pneumonia (0.5\%), } \\
\text { - Unspecified cause }(0.5 \%)\end{array}$ \\
\hline & & $\begin{array}{l}\text { Placebo }+ \\
\text { carboplatin }+ \\
\text { etoposide }\end{array}$ & 196 & $92.3 \%$ & $57.6 \%$ & $24.5 \%$ & $2.5 \%$ & $\begin{array}{l}\text { - IRAEs: } \\
\text { - } \text { Rash (10.2\%), } \\
\text { - Hypothyroidism (0.5\%), } \\
\text { - Hepatitis (4.6\%), } \\
\text { - Infusion-related reaction } \\
\text { - }(5.1 \%), \\
\text { - } \text { Hyperthyroidism }(2.6 \%), \\
\text { - Pneumonitis }(2.6 \%), \\
\text { - } \text { Pancreatitis }(1.0 \%), \\
\text { - } \text { Adrenal insufficiency (1.0\%), } \\
\text { - } \text { Vasculitis }(0.5 \%),\end{array}$ & $\begin{array}{l}\text { - IRAEs: } \\
\text { - Infusion-related reaction } \\
\text { ( } 0.5 \%), \\
\text { - Pneumonitis }(1.0 \%), \\
\text { pancreatitis(1.0\%) }\end{array}$ & $\begin{array}{l}\text { - Pneumonia }(0.5 \%) \text {, } \\
\text { - Septic shock }(0.5 \%) \text {, } \\
\text { - Cardiopulmonary failure } \\
(0.5 \%)\end{array}$ \\
\hline & $\begin{array}{l}\text { IFCT-1603 } \\
\text { (NCT03059667) }\end{array}$ & $\begin{array}{l}\text { Atezolizumab } \\
1,200 \mathrm{mg}\end{array}$ & 48 & NA & NA & $22.9 \%$ & NA & $\begin{array}{l}\text { - IRAEs: } \\
\text { - Hepatitis (4.2\%), } \\
\text { - } \text { Colitis (4.2\%), } \\
\text { - } \text { Arthralgia (6.3\%), } \\
\text { - } \text { Dysthyroidism (4.2\%) }\end{array}$ & NA & None \\
\hline & & Chemotherapy & 24 & NA & NA & NA & NA & NA & NA & None \\
\hline
\end{tabular}


TABLE 3 | Continued

\begin{tabular}{|c|c|c|c|c|c|c|c|c|c|c|}
\hline Target & NCT number & Treatment & $\begin{array}{l}\text { Enrolled } \\
\text { number }\end{array}$ & TRAEs & $\begin{array}{l}\text { TRAEs } \\
\text { (grade } \geq 3 \text { ) }\end{array}$ & IRAEs & $\begin{array}{c}\text { IRAEs } \\
\text { (grade } \geq 3 \text { ) }\end{array}$ & Most common TRAEs/IRAEs & TRAEs/IRAEs (grade $\geq 3$ ) & $\begin{array}{l}\text { Death related to } \\
\text { TRAEs/IRAEs }\end{array}$ \\
\hline & $\begin{array}{l}\text { CASPIAN } \\
\text { (NCT03043872) }\end{array}$ & $\begin{array}{l}\text { Durvalumab } \\
1,500 \mathrm{mg}+ \\
\text { etoposide }+ \\
\text { platinum }\end{array}$ & 265 & $89 \%$ & $46 \%$ & $20 \%$ & $5 \%$ & $\begin{array}{l}\text { IRAEs: } \\
\text { - Hypothyroid (9\%), } \\
\text { - Hyperthyroid (5\%), } \\
\text { - } \text { Pneumonitis }(3 \%), \\
\text { - Hepatic events }(3 \%), \\
\text { - } \quad \text { Dermatitis/rash }(2 \%), \\
\text { - } \text { Diarrhoea/colitis }(2 \%)\end{array}$ & $\begin{array}{l}\text { - IRAEs: } \\
\text { - Pneumonitis }(1 \%), \\
\text { - Hepatic events }(2 \%), \\
\text { - } \text { Diarrhoea/colitis }(<1 \%) \text {, } \\
\text { - Type } 1 \text { diabetes mellitus } \\
\text { - }(2 \%), \\
\text { - Pancreatic }(<1 \%)\end{array}$ & $\begin{array}{l}\text { - IRAEs: } \\
\text { - Cardiac arrest }(<1 \%) \text {, } \\
\text { - Dehydration }(<1 \%), \\
\text { - Hepatotoxicity }(<1 \%), \\
\text { - Pancytopenia }(<1 \%) \text {, } \\
\text { sepsis }(<1 \%)\end{array}$ \\
\hline & & $\begin{array}{l}\text { Etoposide }+ \\
\text { platinum }\end{array}$ & 266 & $90 \%$ & $52 \%$ & $3 \%$ & $<1 \%$ & $\begin{array}{l}\text { - IRAEs: } \\
\text { - Hypothyroid (1\%), } \\
\text { - Pneumonitis (1\%), } \\
\text { - } \text { Dermatitis/rash (1\%), } \\
\text { - } \text { Diarrhoea/colitis (<1\%) }\end{array}$ & $\begin{array}{l}\text { - IRAEs: } \\
\text { - Pneumonitis (<1\%) }\end{array}$ & $\begin{array}{l}\text { - Pancytopenia }(<1 \%) \text {, } \\
\text { - Thrombocytopenia/ } \\
\text { haemorrhage }(<1 \%)\end{array}$ \\
\hline & Goldman et al. (36) & $\begin{array}{l}\text { Durvalumab } \\
10 \mathrm{mg} / \mathrm{kg}\end{array}$ & 21 & $33 \%$ & $0 \%$ & NA & NA & $\begin{array}{l}\text { - TRAEs: } \\
\text { - Nausea (9.5\%), } \\
\text { - Fatigue (9.5\%), } \\
\text { - Rash maculo-papular (9.5\%) }\end{array}$ & None & None \\
\hline \multirow[t]{4}{*}{$\begin{array}{l}\text { CTLA- } \\
4\end{array}$} & $\begin{array}{l}\text { CA184-041 } \\
\text { (NCT00527735) }\end{array}$ & $\begin{array}{l}\text { Placebo/paclitaxel/ } \\
\text { carboplatin }\end{array}$ & 44 & $91 \%$ & $30 \%$ & NA & $9 \%$ & $\begin{array}{l}\text { - TRAEs: } \\
\text { - Rash (4.5\%), } \\
\text { - Pruritus (11.4\%), } \\
\text { - } \text { Diarrhea (25\%) }\end{array}$ & $\begin{array}{l}\text { - TRAEs: } \\
\text { - Diarrhea (11.3\%) }\end{array}$ & None \\
\hline & & $\begin{array}{l}\text { Ipilimumab } \\
10 \mathrm{mg} / \mathrm{kg} / \text { placebo } \\
+ \\
\text { paclitaxel/carboplatin } \\
\text { (concurrent) }\end{array}$ & 42 & $84 \%$ & $43 \%$ & NA & $21 \%$ & $\begin{array}{l}\text { - } \text { TRAEs: } \\
\text { - } \text { rash }(73.8 \%), \\
\text { - } \text { pruritus }(57.1 \%), \\
\text { - } \text { diarrhean }(50 \%)\end{array}$ & $\begin{array}{l}\text { - } \text { TRAEs: } \\
\text { - } \text { Diarrhea (9.5\%), } \\
\text { - } \text { ALT increases (16.7\%), } \\
\text { - } \text { AST increase (11.9\%), } \\
\text { - Hepatitis (2\%) }\end{array}$ & Hepatotoxicity (2.4\%) \\
\hline & & $\begin{array}{l}\text { Ipilimumab } \\
10 \mathrm{mg} / \mathrm{kg} / \text { placebo } \\
+ \\
\text { paclitaxel/carboplatin } \\
\text { (phased) }\end{array}$ & 42 & $95 \%$ & $50 \%$ & NA & $17 \%$ & $\begin{array}{l}\text { - TRAEs: } \\
\text { - Rash (57.1\%), } \\
\text { - Pruritus }(40.4 \%), \\
\text { - Diarrhea }(57.1 \%)\end{array}$ & $\begin{array}{l}\text { - } \text { TRAEs: } \\
\text { - } \text { Diarrhean(23.8\%), } \\
\text { - } \text { Colitis }(2.38 \%), \\
\text { - } \text { Arthralgia }(9.52 \%), \\
\text { - } \text { ALT increases }(4.76 \%), \\
\text { - } \text { AST increases }(7.14 \%), \\
\text { - } \quad \text { Hepatitis }(2 \%)\end{array}$ & None \\
\hline & NCT01331525 & $\begin{array}{l}\text { Ipilimumab } \\
10 \mathrm{mg} / \mathrm{kg}+ \\
\text { carboplatin }+ \\
\text { etoposide }\end{array}$ & 39 & $100 \%$ & $89.7 \%$ & NA & NA & $\begin{array}{l}\text { - IRAEs: } \\
\text { - Diarrhea (72\%), } \\
\text { - Skin rash (51\%) }\end{array}$ & $\begin{array}{l}\text { - IRAEs: } \\
\text { - Ipilimumab related } \\
\text { neurological adverse } \\
\text { events }(7.6 \%)\end{array}$ & $\begin{array}{l}\text { - } \text { Cardiac arrest (2.56\%), } \\
\text { - } \text { Neutropenic sepsis } \\
(2.56 \%), \\
\text { - } \text { Pneumonia (2.56\%), } \\
\text { - } \text { Autoimmune encephalitis } \\
\text { ( } 2.56 \%), \\
\text { - Sepsis }(2.56 \%)\end{array}$ \\
\hline
\end{tabular}


TABLE 3 | Continued

\begin{tabular}{|c|c|c|c|c|c|c|c|c|c|c|}
\hline Target & NCT number & Treatment & $\begin{array}{l}\text { Enrolled } \\
\text { number }\end{array}$ & TRAEs & $\begin{array}{c}\text { TRAEs } \\
\text { (grade } \geq 3 \text { ) }\end{array}$ & IRAEs & $\begin{array}{l}\text { IRAEs } \\
\text { (grade } \geq 3 \text { ) }\end{array}$ & Most common TRAEs/IRAEs & TRAEs/IRAEs (grade $\geq 3$ ) & $\begin{array}{l}\text { Death related to } \\
\text { TRAEs/IRAEs }\end{array}$ \\
\hline & NCT01450761 & $\begin{array}{l}\text { Ipilimumab } \\
10 \mathrm{mg} / \mathrm{kg}+ \\
\text { etoposide }+ \\
\text { platinum (cisplatin } \\
+ \text { carboplatin) }\end{array}$ & 478 & $82 \%$ & $48 \%$ & $57 \%$ & $20 \%$ & $\begin{array}{l}\text { - IRAEs: } \\
\text { - Diarrhea (25\%), } \\
\text { - Rash (19\%), } \\
\text { - Pruritus (12\%), } \\
\text { - Colitis (6\%), alopecia (5\%) }\end{array}$ & $\begin{array}{l}\text { - IRAEs: } \\
\text { - Rash (2\%), } \\
\text { - } \text { Pruritus (1\%), } \\
\text { - } \text { Diarrhea (7\%), } \\
\text { - Colitis (4\%), } \\
\text { - } \text { ALT increased (1\%), } \\
\text { - } \text { AST increased (1\%) }\end{array}$ & $\begin{array}{l}\text { - Colitis }(0.42 \%), \\
\text { - Liver toxicity (0.21\%) }\end{array}$ \\
\hline & & $\begin{array}{l}\text { Placebo + } \\
\text { etoposide + } \\
\text { platinum (cisplatin } \\
+ \text { carboplatin) }\end{array}$ & 476 & $76 \%$ & $44 \%$ & $28 \%$ & $2 \%$ & $\begin{array}{l}\text { - IRAEs: } \\
\text { - Diarrhea (10\%), } \\
\text { - Rash (3\%), } \\
\text { - Pruritus ( } 2 \%), \\
\text { - Colitis (1\%), } \\
\text { - Alopecia (7\%) }\end{array}$ & $\begin{array}{l}\text { - IRAEs: } \\
\text { - Diarrhea (1\%) }\end{array}$ & $\begin{array}{l}\text { - Sepsis }(0.21 \%) \text {, } \\
\text { - Bone marrow } \\
\text { suppression }(0.21 \%)\end{array}$ \\
\hline \multirow[t]{4}{*}{$\begin{array}{l}\text { Double } \\
\text { ICls }\end{array}$} & $\begin{array}{l}\text { CheckMate } 451 \\
\text { (NCT02538666) }\end{array}$ & $\begin{array}{l}\text { Nivolumab } \\
1 \mathrm{mg} / \mathrm{kg}+ \\
\text { ipilimumab } \\
3 \mathrm{mg} / \mathrm{kg}\end{array}$ & 278 & $86 \%$ & $52 \%$ & NA & NA & NA & NA & NA \\
\hline & & $\begin{array}{l}\text { Nivolumab } \\
1 \mathrm{mg} / \mathrm{kg}\end{array}$ & 279 & $61 \%$ & $12 \%$ & NA & NA & NA & NA & NA \\
\hline & & Placebo & 273 & $50 \%$ & $8 \%$ & NA & NA & NA & NA & NA \\
\hline & CheckMate032 & (NCT01928394) & $\begin{array}{l}\text { Nivolumab } \\
3 \mathrm{mg} / \mathrm{kg}\end{array}$ & 98 & $53 \%$ & $13 \%$ & NA & NA & $\begin{array}{l}\text { - } \text { TRAEs: } \\
\text { - } \text { Fatigue }(11 \%), \\
\text { - Pruritus (11\%), } \\
\text { - } \text { Diarrhoea }(7 \%), \\
\text { - } \text { Nausea (7\%), } \\
\text { - } \text { Decreased appetite (6\%), } \\
\text { - Pneumonitis (3\%), } \\
\text { - Vomiting (3\%), } \\
\text { - Hypothyroidism (3\%), } \\
\text { - Hyperthyroidism (2\%), } \\
\text { - } \text { Rash }(2 \%) \text { All <1\% }\end{array}$ & None \\
\hline
\end{tabular}


TABLE 3 | Continued

\begin{tabular}{|c|c|c|c|c|c|c|c|c|c|c|}
\hline Target & NCT number & Treatment & $\begin{array}{l}\text { Enrolled } \\
\text { number }\end{array}$ & TRAEs & $\begin{array}{l}\text { TRAEs } \\
\text { (grade } \geq 3 \text { ) }\end{array}$ & IRAEs & $\begin{array}{l}\text { IRAEs } \\
\text { (grade } \geq 3 \text { ) }\end{array}$ & Most common TRAEs/IRAEs & TRAEs/IRAEs (grade $\geq 3$ ) & $\begin{array}{l}\text { Death related to } \\
\text { TRAEs/IRAEs }\end{array}$ \\
\hline & & $\begin{array}{l}\text { Nivolumab } \\
1 \mathrm{mg} / \mathrm{kg}+ \\
\text { ipilimumab } \\
3 \mathrm{mg} / \mathrm{kg}\end{array}$ & 61 & $79 \%$ & $30 \%$ & NA & NA & $\begin{array}{l}\text { - TRAEs: } \\
\text { - } \text { Fatigue }(26 \%), \\
\text { - Pruritus }(20 \%), \\
\text { - } \text { Diarrhoea (21\%), } \\
\text { - Nausea (12\%), } \\
\text { - } \text { Decreased appetite }(7 \%), \\
\text { - Pneumonitis }(3 \%), \\
\text { - Vomiting (5\%), } \\
\text { - Hypothyroidism (17\%), } \\
\text { - Hyperthyroidism (11\%), } \\
\text { - } \text { Rash (19\%) }\end{array}$ & $\begin{array}{l}\text { - TRAEs: } \\
\text { - Increased lipase (9\%), } \\
\text { - Diarrhoea (5\%) }\end{array}$ & $\begin{array}{l}\text { - Myasthenia gravis }(2 \%) \text {, } \\
\text { - Worsening of renal failure } \\
(2 \%)\end{array}$ \\
\hline & & $\begin{array}{l}\text { Nivolumab } \\
3 \mathrm{mg} / \mathrm{kg}+ \\
\text { ipilimumab } \\
1 \mathrm{mg} / \mathrm{kg}\end{array}$ & 54 & $75 \%$ & $19 \%$ & NA & NA & $\begin{array}{l}\text { - TRAEs: } \\
\text { - Fatigue (22\%), } \\
\text { - } \text { Pruritus (9\%), } \\
\text { - Diarrhoea (17\%), } \\
\text { - Nausea (7\%), } \\
\text { - Decreased appetite (11\%), } \\
\text { - Preumonitis (6\%), } \\
\text { - Vomiting (9\%), } \\
\text { - Hypothyroidism (7\%), } \\
\text { - Hyperthyroidism (6\%), } \\
\text { - Rash (7\%) }\end{array}$ & $\begin{array}{l}\text { - TRAEs: } \\
\text { - Dyspnoea (4\%) }\end{array}$ & Pneumonitis (1\%) \\
\hline & NCT02261220 & $\begin{array}{l}\text { Durvalumab } \\
20 \mathrm{mg} / \mathrm{kg}+ \\
\text { tremelimumab } \\
1 \mathrm{mg} / \mathrm{kg}\end{array}$ & 30 & $67 \%$ & $23 \%$ & NA & NA & $\begin{array}{l}\text { - TRAEs: Fatigue (23\%), } \\
\text { Pruritus (23\%) }\end{array}$ & NA & NA \\
\hline & $\begin{array}{l}\text { BALTIC } \\
\text { (NCT02937818) }\end{array}$ & $\begin{array}{l}\text { Durvalumab } \\
1,500 \text { mg }+ \\
\text { tremelimumab } \\
75 \mathrm{mg}\end{array}$ & 25 & NA & $19 \%$ & NA & NA & NA & NA & NA \\
\hline & $\begin{array}{l}\text { CASPIAN } \\
\text { (NCT03043872) }\end{array}$ & $\begin{array}{l}\text { Durvalumab } \\
1,500 \text { mg }+ \\
\text { tremelimumab } \\
75 \mathrm{mg}+\text { platinum } \\
+ \text { etoposide }\end{array}$ & 266 & $90 \%$ & $55 \%$ & $36 \%$ & $14 \%$ & $\begin{array}{l}\text { - IRAEs: } \\
\text { - Hypothyroid events }(9 \%) \text {, } \\
\text { - Hyperthyroid events }(8 \%), \\
\text { - Diarrhoea/colitis }(8 \%), \\
\text { - Dermatitis/rash }(7 \%), \\
\text { - Hepatic events }(4 \%), \\
\text { - Pneumonitis }(3 \%)\end{array}$ & $\begin{array}{l}\text { - IRAEs: } \\
\text { - Diarrhoea/colitis (3\%), } \\
\text { - Dermatitis/rash (2\%), } \\
\text { - Hepatic events (3\%), }\end{array}$ & $\begin{array}{l}\text { - Enterocolitis }(0.5 \%) \text {, } \\
\text { - Pneumonitis }(0.5 \%) \text {, } \\
\text { - Pneumonitis and hepatitis } \\
\text { in the same patient }(0.5 \%)\end{array}$ \\
\hline & & $\begin{array}{l}\text { Durvalumab } \\
1,500 \mathrm{mg}+ \\
\text { platinum }+ \\
\text { etoposide }\end{array}$ & 265 & $89 \%$ & $46 \%$ & $20 \%$ & $5 \%$ & $\begin{array}{l}\text { - IRAEs: } \\
\text { - Hypothyroid events }(9 \%) \text {, } \\
\text { - Hyperthyroid events }(5 \%), \\
\text { - Hepatic events }(3 \%), \\
\text { - Pneumonitis }(3 \%)\end{array}$ & $\begin{array}{l}\text { - IRAEs: } \\
\text { - Diarrhoea/colitis (1\%), } \\
\text { - Type } 1 \text { diabetes mellitus } \\
\text { (2\%), }\end{array}$ & $\begin{array}{l}\text { - Hepatotoxicity }(0.5 \%) \text {, } \\
\text { - Interstitial lung disease } \\
(0.5 \%)\end{array}$ \\
\hline & & $\begin{array}{l}\text { Platinum + } \\
\text { etoposide }\end{array}$ & 266 & $90 \%$ & $52 \%$ & $3 \%$ & $<1 \%$ & $\begin{array}{l}\text { - IRAEs: } \\
\text { - Hypothyroid events (1\%), } \\
\text { - Diarrhoea/colitis (1\%), } \\
\text { - Pneumonitis (1\%) }\end{array}$ & $\begin{array}{l}\text { - IRAEs: } \\
\text { - Pneumonitis (<1\%) }\end{array}$ & Pneumonitis (<1\%) \\
\hline
\end{tabular}


pembrolizumab as third-line therapy presented the tolerable response for relapsed SCLC. Pembrolizumab combined with thoracic radiotherapy or concurrent chemoradiation therapy presented a good degree of tolerance in preliminary findings. Pembrolizumab combined with platinum-etoposide as the firstline therapy for ES-SCLC patients improved PFS, but the significance threshold for OS was not reached. In contrast, nivolumab monotherapy and pembrolizumab combined with chemotherapy were all not superior to chemotherapy as secondline therapy in recurrent SCLC. Another anti-PD1, tislelizumab plus platinum-etoposide, presented a higher ORR for Chinese ES-SCLC patients, but the result needs to be validated in further studies with large sample sizes. Anti-PD-L1 inhibitors atezolizumab and durvalumab both improved the survival benefits of chemotherapy for SCLC patients, but atezolizumab monotherapy or durvalumab monotherapy failed in secondline therapy for refractory SCLC patients. The results of CTLA4 inhibitors were also dismal. Ipilimumab monotherapy or combined with chemotherapy did not exhibit significant efficacy for newly diagnosed ES-SCLC patients and refractory ES-SCLC patients. Moreover, existing studies could not affirm the efficacy of the combined checkpoint inhibitors in SCLC, as the results of the durvalumab and tremelimumab arm of the CASPIAN study are still pending. However, the double checkpoint inhibitors increased the risk of irAEs. The overall irAEs' occurrence rate in patients with SCLC ranged from 20\% (CASPIAN) to 57\% (NCT01450761) (Table 3). The most commonly reported irAEs were rash, diarrhea, hypothyroidism/hyperthyroidism, colitis, and pneumonia. In addition, nephritis, hepatitis, pancreatitis, and some nervous system-related irAEs were observed. The rate of high grade (grade $\geq 3$ ) irAEs was less than $10 \%$ in most trials, and most irAEs were manageable through systematic therapy in most studies. Pneumonitis was the most frequently reported death-related irAE. Hypothyroidism and hyperthyroidism are reported relatively less frequently in CTLA4 inhibitors compared with PD-1/PD-L1 inhibitors, which was consistent with the findings of a previous study (67). No special safety data were reported. To determine different rates and types of irAEs in SCLC, we compared irAEs reported in other cancer type cohorts from the Checkmate 032, Keynote 028, Keynote 158, NCT01375842 and NCT03432598 (Supplementary Table 1) studies; however, no specific irAEs of SCLC were found. Furthermore, it is difficult to further quantify and compare these indicators because of the variations between studies in terms of the length of median follow-up. Moreover, irAEs in most trials were evaluated by the investigators, which might not be objective and could be lacking a uniform standard. Some studies only reported TRAEs instead of irAEs, and the details of irAEs in most trials are unavailable.

In the 23 trials included (Table 2) in this review, the most commonly reported irAEs/TRAEs were mild. Pneumonitis was the most frequently reported death-related irAE. Other deathrelated irAEs include colitis/intestinal ischemia, encephalopathy, neutropenic sepsis, cardiopulmonary failure, hepatotoxicity, myasthenia gravis, worsening of renal failure, sepsis, and septic shock. Nevertheless, these only account a tiny proportion of irAEs, usually less than $5 \%$. Serious irAEs were the indicator for ICI reduction or discontinuation in most studies, but the treatment details of irAEs were not described.

Some guidelines have been published for the diagnosis and management of irAEs $(68,69)$. IrAEs are graded according to the Common Terminology Criteria for Adverse Events. Mild irAEs graded 1 or 2 could gradually disappear after the discontinuation of ICIs. Moreover, the early identification is of great importance for the management of irAEs. Serious irAEs (grade $\geq 3$ ) threaten the patients' life, corticosteroid therapy was usually needed, and the associated complications, such as infection, were also a source of concern. During the period of clinical therapy, the irAEs had greater complexity, hence individualized treatment and management strategies could be a future research direction.

Furthermore, the study areas of irAEs in SCLC patients that should be addressed are as follows: [1] peculiar irAEs, such as Fanconi syndrome, which was reported in an ES-SCLC patient after he received nivolumab plus ipilimumab as second-line therapy (70); [2] the occurrence rate of irAEs, as this was higher in a real-world report (71); [3] the difference in irAEs between SCLC and NSCLC, as a previous meta-analysis has reported that the occurrence of ICI-related TRAEs in SCLC patients was higher than that in NSCLC patients (72); [4] irAEs of other ICI agents and combination therapies, as new target ICI agents and combination strategies are emerging in SCLC (73); and [5] irAEs of specific populations, as patients with autoimmune diseases are usually excluded from clinical trials, but many patients with SCLC experience paraneoplastic syndromes, and therefore the advantages or disadvantages of ICIs for these populations should be explored in future studies.

\section{CONCLUSION AND PROSPECTS}

The current review summarizes the efficacy and safety data of ICIs in all existing clinical trials in the SCLC treatment field. ICI agents generally demonstrate a promising clinical activity in SCLC therapy, with manageable irAEs, although more detailed data are required. Future study directions include finding reliable biomarkers for the selection of patients that will most benefit from therapy, and verifying the rationale of various combination therapeutic regimens. Moreover, further details regarding irAEs are encouraged to be record and reported in future investigations, which could be of great significance for clinical practice and would benefit the increasing number of patients with SCLC.

\section{AUTHOR CONTRIBUTIONS}

$\mathrm{WH}, \mathrm{XZ}, \mathrm{CY}$, and HZ: conception and design and collection and assembly of data. CY: administrative support. CY and HZ: provision of study materials or patients. WH and XZ: manuscript writing. All authors final approval of manuscript.

\section{SUPPLEMENTARY MATERIAL}

The Supplementary Material for this article can be found online at: https://www.frontiersin.org/articles/10.3389/fonc. 2021.604227/full\#supplementary-material 


\section{REFERENCES}

1. Bray F, Ferlay J, Soerjomataram I, Siegel RL, Torre LA, Jemal A. Global cancer statistics 2018: GLOBOCAN estimates of incidence and mortality worldwide for 36 cancers in 185 countries. CA. (2018) 68:394-424. doi: $10.3322 /$ caac. 21492

2. Semenova E, Nagel R, Berns A. Origins, genetic landscape, and emerging therapies of small cell lung cancer. Genes Dev. (2015) 29:1447-62. doi: 10.1101/gad.263145.115

3. Calles A, Aguado G, Sandoval C, Álvarez R. The role of immunotherapy in small cell lung cancer. Clin Translat Oncol. (2019) 21:961-76. doi: 10.1007/s12094-018-02011-9

4. Zimmerman S, Das A, Wang S, Julian R, Gandhi L, Wolf J. 2017-2018 scientific advances in thoracic oncology: small cell lung cancer. $J$ Thoracic Oncol. (2019) 14:768-83. doi: 10.1016/j.jtho.2019.01.022

5. Van Meerbeeck JP, Fennell DA, De Ruysscher DKM. Small-cell lung cancer. Lancet. (2011) 378:1741-55. doi: 10.1016/S0140-6736(11)60165-7

6. Simeone E, Grimaldi A, Festino L, Trojaniello C, Vitale M, Vanella V, et al. Nivolumab for the treatment of small cell lung cancer. Exp Rev Respirat Med. (2020) 14:5-13. doi: 10.1080/17476348.2020.1681977

7. Eckardt JR, Joachim VP, Jean-Louis P, Zsolt P, Elisabeth Q, Andrea A, et al. Phase III study of oral compared with intravenous topotecan as second-line therapy in small-cell lung cancer. J Clin Oncol Official. (2007) 25:2086-92. doi: 10.1200/JCO.2006.08.3998

8. Owonikoko TK, Behera M, Chen Z, Bhimani C, Ramalingam SS. Metaanalysis of second line chemotherapy efficacy in sensitive and refractory small cell lung cancer (SCLC) patients. J Thoracic Oncol. (2010) 5:S551-2. doi: 10.1097/JTO.0b013e31824c7f4b

9. Facchinetti F, Di Maio M, Tiseo M. Adding PD-1/PD-L1 inhibitors to chemotherapy for the first-line treatment of extensive stage small cell lung cancer (SCLC): a meta-analysis of randomized trials. Cancers. (2020) 12:2645. doi: $10.3390 /$ cancers 12092645

10. Wang B, Xiao B, Li P, Kuang B, Chen W, Li P, et al. Efficacy and safety of first-line immunotherapy in combination with chemotherapy for patients with extensive-stage small cell lung cancer: a systematic review and network meta-analysis. J Oncol. (2020) 2020:2368164. doi: 10.1155/2020/2368164

11. Zhou T, Zhang Z, Luo F, Zhao Y, Hou X, Liu T, et al. Comparison of first-line treatments for patients with extensive-stage small cell lung cancer: a systematic review and network meta-analysis. JAMA Netw Open. (2020) 3:e2015748. doi: 10.1001/jamanetworkopen.2020. 15748

12. Sławiński G, Wrona A, Dabrowska-Kugacka A, Raczak G, Lewicka E. Immune checkpoint inhibitors and cardiac toxicity in patients treated for non-small lung cancer: a review. Int J Mol Sci. (2020) 21:7195. doi: 10.3390/ijms21197195

13. Liu YH, Zang XY, Wang JC, Huang SS, Xu J, Zhang P. Diagnosis and management of immune related adverse events (irAEs) in cancer immunotherapy. Biomed Pharmacother. (2019) 120:109437. doi: 10.1016/j.biopha.2019.109437

14. Friedman CF, Proverbs-Singh TA, Postow MA. Treatment of the immunerelated adverse effects of immune checkpoint inhibitors: a review. JAMA Oncol. (2016) 2:1346-53. doi: 10.1001/jamaoncol.2016.1051

15. Park R, Lopes L, Saeed A. Anti-PD-1/L1-associated immune-related adverse events as harbinger of favorable clinical outcome: systematic review and meta-analysis. Clin Transl Oncol. (2020) 23:100-9. doi: 10.1007/s12094-020-02397-5

16. Shimozaki K, Sukawa Y, Beppu N, Kurihara I, Suzuki S, Mizuno R, et al. Multiple immune-related adverse events and anti-tumor efficacy: real-world data on various solid tumors. Cancer Manage Res. (2020) 12:4585-93. doi: 10.2147/CMAR.S247554

17. Iams WT, Porter J, Horn L. Immunotherapeutic approaches for small-cell lung cancer. Nat Rev Clin Oncol. (2020) 17:300-12. doi: 10.1038/s41571-0190316-z

18. Quandt D, Hoff H, Rudolph M, Fillatreau S, Brunner-Weinzierl MC. A new role of CTLA-4 on B cells in thymus-dependent immune responses in vivo. J Immunol. (2007) 179:7316-24. doi: 10.4049/jimmunol.179.11.7316

19. Schreiber RD, Old LJ, Smyth MJ. Cancer immunoediting: integrating immunity's roles in cancer suppression and promotion. Science. (2011) 331:1565-70. doi: 10.1126/science. 1203486
20. Sznol M, Chen L. Antagonist antibodies to PD-1 and B7-H1 (PD-L1) in the treatment of advanced human cancer-response. Clin Cancer Res. (2013) 19:5542. doi: 10.1158/1078-0432.CCR-13-2234

21. Zhou X, Hou W, Gao L, Shui L, Yi C, Zhu H. Synergies of antiangiogenic therapy and immune checkpoint blockade in renal cell carcinoma: from theoretical background to clinical reality. Front Oncol. (2020) 10:1321. doi: $10.3389 /$ fonc. 2020.01321

22. Longo DL, Postow MA, Sidlow R, Hellmann MD. Immune-related adverse events associated with immune checkpoint blockade. N Engl J Med. (2018) 378:158-68. doi: 10.1056/NEJMra1703481

23. Byrne EH, Fisher DE. Immune and molecular correlates in melanoma treated with immune checkpoint blockade. Cancer. (2017) 123:2143. doi: $10.1002 / \mathrm{cncr} .30444$

24. Callahan MK, Yang A, Tandon S. Evaluation of serum IL-17 levels during ipilimumab therapy: correlation with colitis. J Clin Oncol. (2011) 29:319-21. doi: 10.1200/jco.2011.29.15_suppl.2505

25. Fan Y, Geng Y, Shen L, Zhang Z. Advances on immune-related adverse events associated with immune checkpoint inhibitors. Front Med. (2020) 15:33-42. doi: 10.1007/s11684-019-0735-3

26. Mangan B, McAlister R, Balko J, Johnson D, Moslehi J, Gibson A, et al. Evolving insights into the mechanisms of toxicity associated with immune checkpoint inhibitor therapy. Br J Clin Pharmacol. (2020) 86:1778-89. doi: 10.1111/bcp.14433

27. Alissafi T, Hatzioannou A, Legaki A, Varveri A, Verginis P. Balancing cancer immunotherapy and immune-related adverse events: the emerging role of regulatory T cells. J Autoimmunity. (2019) 104:102310. doi: 10.1016/j.jaut.2019.102310

28. Chalmers Z, Connelly C, Fabrizio D, Gay L, Ali S, Ennis R, et al. Analysis of 100,000 human cancer genomes reveals the landscape of tumor mutational burden. Genome Med. (2017) 9:34. doi: 10.1186/s13073-017-0424-2

29. McGranahan N, Furness A, Rosenthal R, Ramskov S, Lyngaa R, Saini S, et al. Clonal neoantigens elicit $\mathrm{T}$ cell immunoreactivity and sensitivity to immune checkpoint blockade. Science. (2016) 351:1463-9. doi: 10.1126/science.aaf1490

30. Reck M, Schenker M, Lee K, Provencio M, Nishio M, Lesniewski-Kmak K, et al. Nivolumab plus ipilimumab versus chemotherapy as first-line treatment in advanced non-small-cell lung cancer with high tumour mutational burden: patient-reported outcomes results from the randomised, openlabel, phase III CheckMate 227 trial. Eur J Cancer. (2019) 116:137-47. doi: 10.1016/j.ejca.2019.05.008

31. Rizvi N, Hellmann M, Snyder A, Kvistborg P, Makarov V, Havel J, et al. Cancer immunology. Mutational landscape determines sensitivity to PD1 blockade in non-small cell lung cancer. Science. (2015) 348:124-8. doi: $10.1126 /$ science.aaal 348

32. Schumacher TN, Schreiber RD. Neoantigens in cancer immunotherapy. Science. (2015) 348:69-74. doi: 10.1126/science.aaa4971

33. Iams WT, Shiuan E, Meador CB, Roth M, Bordeaux J, Vaupel C, et al. Improved prognosis and increased tumor-infiltrating lymphocytes in patients who have SCLC with neurologic. J Thorac Oncol. (2019) 14:1970-81. doi: $10.1016 /$ j.jtho.2019.05.042

34. Maddison P, Newsom-Davis J, Mills K, Souhami R. Favourable prognosis in Lambert-Eaton myasthenic syndrome and small-cell lung carcinoma. Lancet. (1999) 353:117-8. doi: 10.1016/S0140-6736(05)76153-5

35. Reck M, Vicente D, Ciuleanu T, Gettinger S, Peters S, Horn L, et al. LBA5 Efficacy and safety of nivolumab (nivo) monotherapy versus chemotherapy (chemo) in recurrent small cell lung cancer (SCLC): results from CheckMate 331. Annals Oncol. (2018) 29:43. doi: 10.1093/annonc/mdy511.004

36. Goldman JW, Dowlati A, Antonia SJ, Nemunaitis JJ, Gadgeel SM. Safety and antitumor activity of durvalumab monotherapy in patients with pretreated extensive disease small-cell lung cancer (ED-SCLC). J Clin Oncol. (2018) 36:8518. doi: 10.1200/JCO.2018.36.15_suppl.8518

37. Cai H, Zhang H, Jiang Y. Prognostic and clinicopathological value of programmed cell death ligand1 expression in patients with small cell lung cancer: a meta-analysis. Front Oncol. (2020) 10:1079. doi: $10.3389 /$ fonc. 2020.01079

38. Melosky B, Cheema P, Brade A, McLeod D, Liu G, Price P, et al. Prolonging survival: the role of immune checkpoint inhibitors in the treatment of extensive-stage small cell lung cancer. Oncologist. (2020) 25:981-92. doi: 10.1634/theoncologist.2020-0193 
39. Reddy H, Qin A, Kalemkerian G. Emerging drugs for small cell lung cancer: a focused review on immune checkpoint inhibitors. Exp Opin Emerg Drugs. (2020) 25:353-66. doi: 10.1080/14728214.2020.1798929

40. Mansfield A, Kazarnowicz A, Karaseva N, Sanchez A, De Boer R, Andric Z, et al. Safety and patient-reported outcomes of atezolizumab, carboplatin, and etoposide in extensive-stage small-cell lung cancer (IMpower133): a randomized phase I/III trial. Annals Oncol. (2020) 31:310-7. doi: 10.1016/j.annonc.2019.10.021

41. Huang W, Chen J, Xing R, Zeng Y. Combination therapy: Future directions of immunotherapy in small cell lung cancer. Translat Oncol. (2020) 14:100889. doi: 10.1016/j.tranon.2020.100889

42. Xue Y, Gao S, Gou J, Yin T, He H, Wang Y, et al. Platinum-based chemotherapy in combination with PD-1/PD-L1 inhibitors: preclinical and clinical studies and mechanism of action. Exp Opin Drug Delivery 2020:1-17. doi: 10.1080/17425247.2021.1825376

43. Ready N, Farago A, de Braud F, Atmaca A, Hellmann M, Schneider J, et al. Third-Line Nivolumab Monotherapy in Recurrent SCLC: checkMate 032. J Thoracic Oncol. (2019) 14:237-44. doi: 10.1016/j.jtho.2018.10.003

44. Fessas P, Lee H, Ikemizu S, Janowitz T. A molecular and preclinical comparison of the PD-1-targeted T-cell checkpoint inhibitors nivolumab and pembrolizumab. Semin Oncol. (2017) 44:136-40. doi: 10.1053/j.seminoncol.2017.06.002

45. Ott PA, Elez E, Hiret S, Kim DW, Morosky A, Saraf S, et al. Pembrolizumab in patients with extensive-stage small-cell lung cancer: results from the phase Ib KEYNOTE-028 study. J Clin Oncol. (2017) 35:3823-9. doi: 10.1200/JCO.2017.72.5069

46. Chung HC, Lopez-Martin JA, Kao CH, Miller WH, Piha-Paul SA. Phase 2 study of pembrolizumab in advanced small-cell lung cancer (SCLC): KEYNOTE-158. J Clin Oncol. (2018) 36:8506. doi: 10.1200/JCO.2018.36.15_suppl.8506

47. Chung H, Piha-Paul S, Lopez-Martin J, Schellens J, Kao S, Miller W, et al. Pembrolizumab after two or more lines of previous therapy in patients with recurrent or metastatic SCLC: results from the KEYNOTE028 and KEYNOTE-158 studies. J Thoracic Oncol. (2020) 15:618-27. doi: 10.1016/j.jtho.2019.12.109

48. Gadgeel S, Pennell N, Fidler M, Halmos B, Bonomi P, Stevenson J, et al. Phase II study of maintenance pembrolizumab in patients with extensivestage small cell lung cancer (SCLC). J Thoracic Oncol. (2018) 13:1393-9. doi: $10.1016 /$ j.jtho.2018.05.002

49. Welsh J, Heymach J, Chen D, Verma V, Cushman T, Hess K, et al. Phase I trial of pembrolizumab and radiation therapy after induction chemotherapy for extensive-stage small cell lung cancer. J Thoracic Oncol. (2020) 15:266-73. doi: 10.1016/j.jtho.2019.10.001

50. Kim YJ, Keam B, Ock CY, Song S, Heo DS. A phase II study of pembrolizumab and paclitaxel in patients with relapsed or refractory small-cell lung cancer. Lung Cancer. (2019) 136:122-8. doi: 10.1016/j.lungcan.2019.08.031

51. Rudin C, Awad M, Navarro A, Gottfried M, Peters S, Csoszi T, et al. Pembrolizumab or placebo plus etoposide and platinum as first-line therapy for extensive-stage small-cell lung cancer: randomized, doubleblind, phase III KEYNOTE-604 study. J Clin Oncol. (2020) 38:2369-79. doi: $10.1200 / J C O .20 .00793$

52. Wang Z, Zhao J, Ma Z, Cui J, Shu Y, Liu Z, et al. A Phase 2 Study of tislelizumab in combination with platinum-based chemotherapy as first-line treatment for advanced lung cancer in chinese patients. Lung Cancer. (2020) 147:259-68. doi: 10.1016/j.lungcan.2020.06.007

53. Sequist LV, Chiang A, Gilbert J, Gordon M, Gettinger S. Clinical activity, safety and predictive biomarkers results from a phase Ia atezolizumab (atezo) trial in extensive-stage small cell lung cancer (ES-SCLC). Annals Oncol. (2016) 27(suppl_6):vi493. doi: 10.1093/annonc/mdw389.03

54. Horn L, Mansfield A, Szczęsna A, Havel L, Krzakowski M, Hochmair M, et al. First-line atezolizumab plus chemotherapy in extensive-stage smallcell lung cancer. N Engl J Med. (2018) 379:2220-9. doi: 10.1056/NEJMoa18 09064

55. Pujol J, Greillier L, Audigier-Valette C, Moro-Sibilot D, Uwer L, Hureaux J, et al. A randomized non-comparative phase II study of anti-programmed cell death-ligand 1 atezolizumab or chemotherapy as second-line therapy in patients with small cell lung cancer: results from the IFCT1603 trial. J Thoracic Oncol. (2019) 14:903-13. doi: 10.1016/j.jtho.2019. 01.008
56. Paz-Ares L, Dvorkin M, Chen Y, Reinmuth N, Hotta K, Trukhin D, et al. Durvalumab plus platinum-etoposide versus platinum-etoposide in first-line treatment of extensive-stage small-cell lung cancer (CASPIAN): a randomised, controlled, open-label, phase 3 trial. Lancet. (2019) 394:1929-39. doi: 10.1016/S0140-6736(19)32222-6

57. Reck M, Bondarenko I, Luft A, Serwatowski P, Barlesi F, Chacko R, et al. Ipilimumab in combination with paclitaxel and carboplatin as firstline therapy in extensive-disease-small-cell lung cancer: results from a randomized, double-blind, multicenter phase 2 trial. Annals Oncol. (2013) 24:75-83. doi: $10.1093 /$ annonc/mds213

58. Arriola E, Wheater M, Galea I, Cross N, Maishman T, Hamid D, et al. Outcome and biomarker analysis from a multicenter phase 2 study of ipilimumab in combination with carboplatin and etoposide as first-line therapy for extensive-stage SCLC. J Thoracic Oncol. (2016) 11:1511-21. doi: 10.1016/j.jtho.2016.05.028

59. Reck M, Luft A, Szczesna A, Havel L, Kim S, Akerley W, et al. Phase III randomized trial of ipilimumab plus etoposide and platinum versus placebo plus etoposide and platinum in extensive-stage small-cell lung cancer. J Clin Oncol. (2016) 34:3740-8. doi: 10.1200/JCO.2016.67.6601

60. Hodi FS, Chiarion-Sileni V, Gonzalez R, Grob J-J, Rutkowski P, Cowey CL, et al. Nivolumab plus ipilimumab or nivolumab alone versus ipilimumab alone in advanced melanoma (CheckMate 067): 4-year outcomes of a multicentre, randomised, phase 3 trial. Lancet Oncol. (2018) 19:1480-92. doi: 10.1016/S1470-2045(18)30700-9

61. Scherpereel A, Mazieres J, Greillier L, Lantuejoul S, Dô P, Bylicki O, et al. Nivolumab or nivolumab plus ipilimumab in patients with relapsed malignant pleural mesothelioma (IFCT-1501 MAPS2): a multicentre, openlabel, randomised, non-comparative, phase 2 trial. Lancet Oncol. (2019) 20:239-53. doi: 10.1016/S1470-2045(18)30765-4

62. Antonia S, López-Martin J, Bendell J, Ott P, Taylor M, Eder J, et al. Nivolumab alone and nivolumab plus ipilimumab in recurrent small-cell lung cancer (CheckMate 032): a multicentre, open-label, phase 1/2 trial. Lancet Oncol. (2016) 17:883-95. doi: 10.1016/S1470-2045(16)30098-5

63. Ready N, Owonikoko TK, Postmus PE, Zhu H. CheckMate 451: a randomized, double-blind, phase III trial of nivolumab (nivo), nivo plus ipilimumab (ipi), or placebo as maintenance therapy in patients (pts) with extensivestage disease small cell lung cancer (ED-SCLC) after first-line platinumbased doublet chemotherapy (PT-DC). J Clin Oncol. (2016) 34(15_Suppl). doi: 10.1200/JCO.2016.34.15_suppl.TPS8579

64. Cho DC, Mahipal A, Dowlati A, Chow WA, Segal NH, Chung KY, et al. Safety and clinical activity of durvalumab in combination with tremelimumab in extensive disease small-cell lung cancer (ED-SCLC). J Clin Oncol. (2018) 36:8517. doi: 10.1200/JCO.2018.36.15_suppl.8517

65. Bondarenko I, Juan-Vidal O, Pajkos G, Kryzhanivska A, Pápai Székely $\mathrm{Z}$, Vicente D, et al. 1665PDPreliminary efficacy of durvalumab plus tremelimumab in platinum-refractory resistant ED-SCLC from arm A of the phase II BALTIC study. Annal Oncol. (2018) 29(suppl_8). doi: 10.1093/annonc/mdy298.001

66. Goldman J, Dvorkin M, Chen Y, Reinmuth N, Hotta K, Trukhin D, et al. Durvalumab, with or without tremelimumab, plus platinum-etoposide versus platinum-etoposide alone in first-line treatment of extensive-stage small-cell lung cancer (CASPIAN): updated results from a randomised, controlled, open-label, phase 3 trial. Lancet Oncol. (2021) 22:51-65. doi: 10.1016/S1470-2045(20)30539-8

67. Del Rivero J, Cordes L, Klubo-Gwiezdzinska J, Madan R, Nieman L, Gulley J. Endocrine-related adverse events related to immune checkpoint inhibitors: proposed algorithms for management. Oncologist. (2020) 25:290300. doi: 10.1634/theoncologist.2018-0470

68. Brahmer JR, Lacchetti C, Schneider BJ, Atkins MB, Thompson JA. Management of immune-related adverse events in patients treated with immune checkpoint inhibitor therapy american society of clinical oncology clinical practice guideline. J Oncol Pract. (2018) 36:JOP1800005. doi: 10.1200/JOP.18.00005

69. Thompson JA, Schneider BJ, Brahmer J, Andrews S, Scavone JL. Management of immunotherapy-related toxicities, version 1.2019, NCCN clinical practice guidelines in oncology. J Natl Comprehens Cancer Netw. (2019) 17:255-89. doi: 10.6004/jncen.2019.0013

70. Farid S, Latif H, Nilubol C, Kim C. Immune checkpoint inhibitor-induced fanconi syndrome. Cureus. (2020) 12:e7686. doi: 10.7759/cureus.7686 
71. Schmid S, Mauti LA, Friedlaender A, Blum V, Früh M. Outcomes with immune checkpoint inhibitors for relapsed small-cell lung cancer in a Swiss cohort. Cancer Immunol Immunother. (2020) 69:1605-13. doi: 10.1007/s00262-020-02565-0

72. Shao J, Wang C, Ren P, Jiang Y, Tian P, Li W. Treatment- and immune-related adverse events of immune checkpoint inhibitors in advanced lung cancer. Biosci Rep. (2020) 40:BSR20192347. doi: 10.1042/BSR20192347

73. Saltos A, Shafique M, Chiappori A. Update on the biology, management, and treatment of small cell lung cancer (SCLC). Front Oncol. (2020) 10:1074. doi: $10.3389 /$ fonc. 2020.01074
Conflict of Interest: The authors declare that the research was conducted in the absence of any commercial or financial relationships that could be construed as a potential conflict of interest.

Copyright (c) $2021 \mathrm{Hou}, \mathrm{Zhou}, \mathrm{Yi}$ and Zhu. This is an open-access article distributed under the terms of the Creative Commons Attribution License (CC BY). The use, distribution or reproduction in other forums is permitted, provided the original author(s) and the copyright owner(s) are credited and that the original publication in this journal is cited, in accordance with accepted academic practice. No use, distribution or reproduction is permitted which does not comply with these terms. 